University of Louisville

ThinkIR: The University of Louisville's Institutional Repository

Electronic Theses and Dissertations

$12-2019$

\title{
Stabilization of bimodal colloidal systems via nanoparticle haloing in microgravity.
}

Luke Hawtrey

University of Louisville

Follow this and additional works at: https://ir.library.louisville.edu/etd

Part of the Complex Fluids Commons, and the Other Chemical Engineering Commons

\section{Recommended Citation}

Hawtrey, Luke, "Stabilization of bimodal colloidal systems via nanoparticle haloing in microgravity." (2019). Electronic Theses and Dissertations. Paper 3356.

https://doi.org/10.18297/etd/3356

This Master's Thesis is brought to you for free and open access by ThinkIR: The University of Louisville's Institutional Repository. It has been accepted for inclusion in Electronic Theses and Dissertations by an authorized administrator of ThinkIR: The University of Louisville's Institutional Repository. This title appears here courtesy of the author, who has retained all other copyrights. For more information, please contact thinkir@louisville.edu. 


\title{
STABILIZATION OF BIMODAL COLLOIDAL SYSTEMS VIA NANOPARTICLE HALOING IN MICROGRAVITY
}

By

Luke Thomas Hawtrey

B.S. University of Louisville 2018

\author{
A Thesis \\ Submitted to Faculty of the \\ J.B. Speed School of Engineering of the University of Louisville \\ For the Degree of \\ Master of Engineering \\ In Chemical Engineering \\ Department of Chemical Engineering \\ University of Louisville \\ Louisville, Kentucky
}

December 2019 

STABILIZATION OF BIMODAL COLLOIDAL SYSTEMS VIA NANOPARTICLE HALOING IN MICROGRAVITY

By

Luke Thomas Hawtrey

B.S. University of Louisville 2018

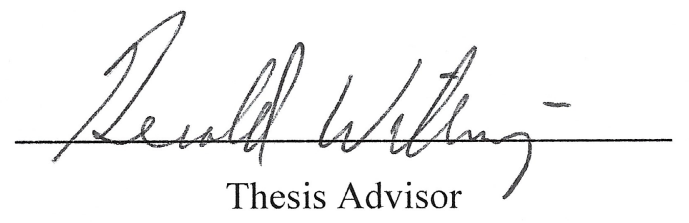

Dr. Gerold Willing

Ste v helen

Dr. Stuart Williams

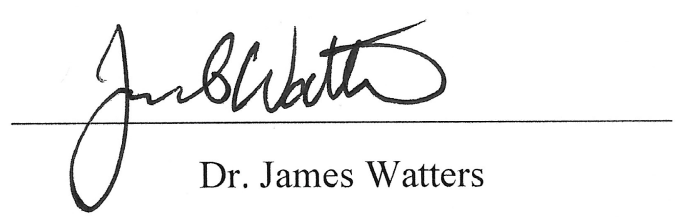




\section{ABSTRACT \\ STABILIZATION OF BIMODAL COLLOIDAL SYSTEMS VIA NANOPARTICLE HALOING IN MICROGRAVITY}

Colloidal suspensions typically contain a multi-phase system of solid particles suspended in a liquid medium. Colloids are widely used in industrial applications such as inks, paints, motor oils, foods, cosmetics, and many more. Colloidal systems are typically formed by the interaction of the attractive van der Waals forces and one or more repulsive forces. These repulsive forces include electrostatic repulsion, steric hindrance, and nanoparticle haloing. Nanoparticle Haloing (NPH) is a phenomenon discovered in 2001 as a viable method to stabilize colloidal systems of uncharged silica microparticles using highly charged zirconia nanoparticles. For this thesis the effects of NPH were tested and compared between ground-based experiments and experiments conducted in microgravity aboard the International Space Station (ISS). This study found that for the same bimodal sample of $0.055 \mathrm{vol} \%$ nanoparticles and $1 \mathrm{vol} \%$ microparticles, the time for the system to reach steady state was 30 minutes in gravity settling conditions versus less than three minutes in a microgravity environment. This indicated that the ground experiments were better mixed due to sonication than the microgravity samples, which used magnetic stir bar mixing.

The data presented herein illustrates the effects of microgravity on the NPH system and the viability of nanoparticle haloing as a stabilization mechanism for future applications such as highperformance quantum-dot solar cells (QDSS). This study notes key factors for colloidal systems in microgravity and lays the groundwork for future nanoparticle haloing experiments. During the experiment potential issues were noted such as illumination, image capture rate, and the mixing 
apparatus. For each issue a recommendation is made for future work in nanoparticle haloing colloidal systems in a microgravity environment. 


\section{ACKNOWLEDGEMENTS}

Firstly, I would like to thank Dr. Willing for taking me onto the nanoparticle haloing project. It is his support and advice that guided me while working on this thesis. I would also like to thank Dr. Williams for his help, particularly during the revision process. He was a massive help during the entire project, especially during the final emendations. My special thanks to my family, who supported and encouraged me throughout my entire academic career. They inspire me even in the difficult hours.

Finally my sincere thanks to the scientists at the NASA Glenn Center and Zin Technologies for their help and recommendations. During the preparation process for this experiment, they offered valuable advice on the ordering and running of the tests. 


\section{TABLE OF CONTENTS}

APPROVAL PAGE. .ii

ABSTRACT .iii

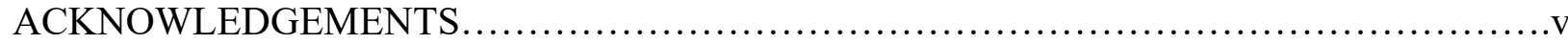

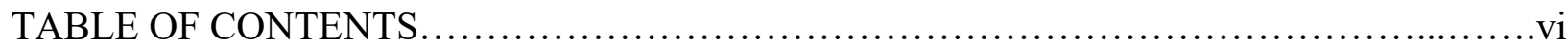

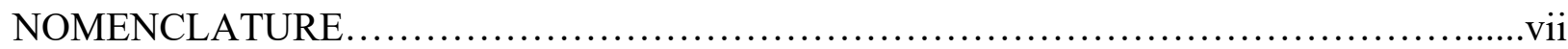

LIST OF FIGURES..........................................................viii

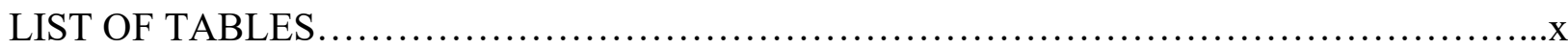

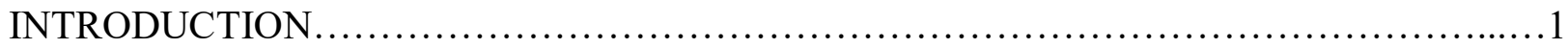

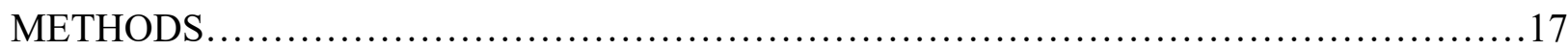

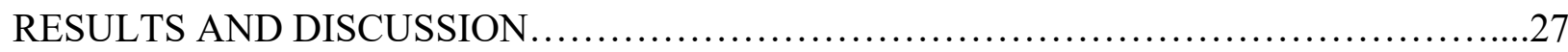

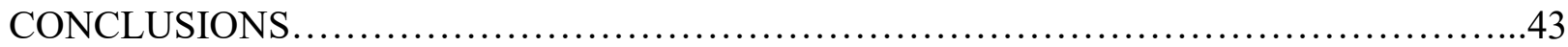

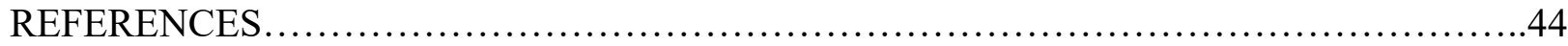

APPENDIX I: ZETA POTENTIAL RESULTS FOR SSQ BATCHES .....................47

APPENDIX II: GROUND BLOB SIZE ANALYSIS RESULTS $\ldots \ldots \ldots \ldots \ldots \ldots \ldots \ldots \ldots \ldots \ldots . . \ldots 49$

APPENDIX III: FULL SOURCE CODE FOR BLOB SIZE ANALYSIS MACRO............58

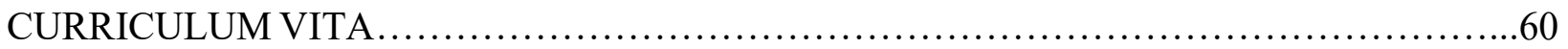




\section{NOMENCLATURE}

$A=$ The Hamaker coefficient $\left(\sim 10^{-19}\right.$ depending on material properties)

$$
C=\text { The potential 2-body interaction parameter }
$$

$\rho_{1}=$ The number density for particle 1

$\rho_{2}=$ The number density for particle 2

$$
F_{v d w}=\text { The Van der Waals Force }
$$

$w(r)=$ The VDW interaction potential

$$
R=\text { Particle radius }
$$

$$
D=\text { Distance between the particles }
$$


LIST OF FIGURES

FIGURE 1: A PHASE DIAGRAM OF ATTRACTIVE MICROSPHERES AND REPULSIVE

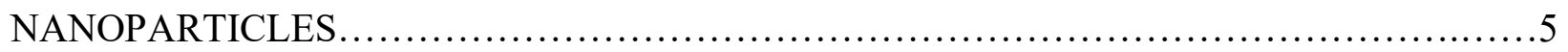

FIGURE 2: AN ILLUSTRATION OF THE DOUBLE-LAYER EFFECT WITHIN A

CHARGED PARTICLE SYSTEM................................................. 9

FIGURE 3: COLLOIDAL STABILIZATION MECHANISMS INCLUDING a)

ELECTROSTATIC b) STERIC AND c) NANOPARTICLE HALOING.......................11

FIGURE 4: THE DESIGN OF AN ATOMIC FORCE MICROSCOPE......................13

FIGURE 5: INTERACTION FORCE ON A SILICA MICROPARTICLE IN A

NANOPARTICLE HALOING SYSTEM........................................... 14

FIGURE 6:COMPONENTS OF THE LIGHT MICROSCOPY MODULE...................16

FIGURE 7: A REPRESENTATIVE UNIT STRUCTURE OF SILSESQUIOXANE............17

FIGURE 8: SYNTHESIS OF BENZYL CHLORIDE SILSESQUIOXANE

MICROPARTICLES ............................................................. 19

FIGURE 9: FUNCTIONALIZATION OF BC-SSQ WITH RHODAMINE B................20

FIGURE 10: A SAMPLE CAPILLARY TUBE WITHIN THE 3D-PRINTED HOLDER.......17

FIGURE 11: NIKON CONFOCAL MICROSCOPE FOR GROUND EXPERIMENTS.........21

FIGURE 12: NPH SAMPLES LOADED INTO THE INTEGRATED IMAGING AND MIXING

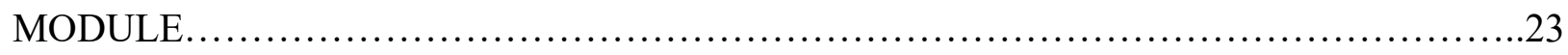


FIGURE 13: ZETA POTENTIAL RESULTS FOR BATCH 12 MP SAMPLES. .25

FIGURE 14: ZETA POTENTIAL RESULTS FOR BATCH 12 RB MP SAMPLES. .26

FIGURE 15: BLOB SIZE RESULTS FOR 0.1\% NANOPARTICLE RUN 2 ..................27

FIGURE 16: BLOB SIZE RESULTS FOR 0.1\% NANOPARTICLE RUN 1 .28

FIGURE 17: BLOB SIZE RESULTS FOR 0.055\% NANOPARTICLE RUN 1

FIGURE 18: BLOB SIZE RESULTS FOR 0.055\% NANOPARTICLE RUN 3 .29

FIGURE 19: BLOB SIZE RESULTS FOR 0.01\% NANOPARTICLE RUN 4. 30

FIGURE 20: A REPRESENTATIVE 20X IMAGE FROM SR1 MICROGRAVITY

IMAGING 31

FIGURE 21: 2.5X IMAGE OF CAPILLARY 1 (LEFT) AND CAPILLARY 3 (RIGHT) SHOWING BUBBLE FORMATION. .31

FIGURE 22: BLOB SIZE RESULTS FOR 0.055\% NANOPARTICLE RUN $1,2,3 \ldots \ldots \ldots \ldots . . .33$

FIGURE 23: NANOPARTICLE ADSORBTION VS. NANOPARTICLE CONCENTRATION. .35

FIGURE 24: DIFFERENCE IN ILLUMINATION BETWEEN SR1 (LEFT) SR3 (RIGHT) AND SR4 (BOTTOM) .37

FIGURE 25: DEVELOPMENT OF FISSURE IN CAPILLARY 2. 39 


\section{LIST OF TABLES}

TABLE I: COLLOIDAL SYSTEMS BY PHASE .....................................

TABLE II: ZETA POTENTIAL VS. STABILITY BEHAVIOR ........................... 10

TABLE III: MIXING AMOUNTS FOR THE COLLOIDAL MIXTURE.....................20 


\section{INTRODUCTION}

\section{A. The Importance of Colloids}

Colloidal suspensions or "colloids" are heterogeneous mixtures in which particles are suspended in a surrounding medium. Colloidal suspensions consist of two phases: a dispersed phase such as a solid particulate and a continuous phase such as a liquid medium which aids in suspension. Colloidal systems may be solid, liquid, or gas, however a common form of colloid used in industry is a solid phase dispersed within a liquid medium.

While colloids take the form of solids suspended in a liquid, they can appear in many forms depending on the phases involved. The variations in colloidal suspensions based on the phases are shown in Table I below.

\section{TABLE I: COLLOIDAL SYSTEMS BY PHASE}

Dispersed Phase

\begin{tabular}{ccccc} 
& \multicolumn{2}{c}{ Gas } & Liquid & Solid \\
\cline { 2 - 5 } Continuous & Gas & None (Gases are soluble) & Aerosol (ex: fog) & Aerosol (ex: plaster) \\
Phase & Liquid & Foams (ex: whipped cream) & Emulsion (ex: egg yolk) & Sol (ex: gelatin) \\
& Solid & Solid foams (ex: memory foam) & Gel (ex: cheese) & Sol (ex: colored glass) \\
\hline
\end{tabular}


This multiphase versatility of colloids allows them to be used for many applications. Colloids can appear in nature, such as in rain or clouds. Many food products are colloids including milk, butter, and whipped cream. Colloids are also widely used in industrial applications, such as inks, paints, motor oils, foods, cosmetics, and many more. Colloids can be used as a thickener or to increase the springiness of a material such as a foam. In ballpoint pens, a liquid solid colloid (a gel) is often used as ink. Due to the wide use and versatility of colloids, many researchers have invested significant time into the study and furthering of colloid science. Colloid science, the study of colloidal systems and their properties, dates to the $19^{\text {th }}$ century. The following describes a brief history of colloidal systems.

\section{B. A Brief History of Colloids}

Colloidal science originated with the Italian chemist and toxicologist Francesco Selmi. In 1845 Selmi first described properties of colloids in his studies of silver chloride, Prussian blue, and sulfur in water, naming the colloids "pseudo solutions" due to their low diffusion rate compared to the diffusion rate of atoms and molecules (Selmi, 1845). The term "Colloid" was first coined in 1861 by the British chemist Thomas Graham. Graham derived the term from the Greek word "kolla" meaning glue to describe Selmi's "pseudo solutions" (Graham, 1861). Further

research showed that this low diffusion rate was in fact caused by their large size compared to atoms, over $1 \mathrm{~nm}$ in diameter.

Some decades before, an English botanist Robert Brown was observing a curious behavior of pollen grains in water. Brown noticed that rather than remaining still, these grains exhibited a permanent motion which was eventually named after him; Brownian motion. This behavior was 
defined as the property of these particles to move in any direction (Brown, 1828). In 1905-1906 Albert Einstein and Marian Smoluchowski produced the first quantitative theory for Brownian motion, confirming the ideas of Robert Brown (Einstein, 1905).

In the latter part of the $20^{\text {th }}$ century it was shown that colloidal behavior in a solvent closely mimicked the behavior of atoms and molecules (A. Vrij, 1978). This behavior allowed for the modeling of molecular systems using the relatively large colloidal systems. Due to their large size, colloidal particles are much easier to observe than their miniature molecular cousins.

Currently colloidal systems build off the work done by Einstein, Brown, Graham, and many more to apply colloids to a wide variety of industrial materials and products. Along with those applications mentioned in the previous section, Colloids are utilized in the medical industry. Many pharmaceuticals (Kreuter, 1994), DNA separations (Gerhard Gompper, 2007), cellular separations (Jelinek, 2009), and in-vitro diagnostics (Hassan Azzazy, 2009) utilize the colloidal science first identified back in the early $19^{\text {th }}$ century. Due to the versatility of colloidal science, researchers are continuously striving to improve the knowledge of and use cases for colloidal systems.

Recent work has been done in the colloidal field on the application of quantum-dot synthesized solar cells (QDSS) (Nozik, 2002). QDSS are a novel type of solar cell that utilizes the unique properties of quantum dots, nanoparticles in the range of 4-10 $\mathrm{nm}$ in diameter. These dots have the property of a tunable band gap, which allows their absorption wavelength to be altered based on their size (Carey et al., 2015). A material's band gap is the minimum energy required to excite an electron from its valence band to its conduction band. Research has been ongoing in the application of quantum dots to photovoltaic cells. By tuning the band gap of the quantum dots, researchers have been able to increase the efficiency of QDSS to $12 \%$. When stacking cells of 
tuned colloidal quantum dots, the limiting efficiency (known as the Shockley-Queisser limit) for these solar cells can be increased. Based on a study published in 2019, the limiting efficiency for a 4-cell PbS QDSS is 52.5\% (Gao et al., 2019). This compares to the $30 \%$ limiting efficiency of a traditional silicon-based solar cell (Rühle, 2016). By using colloidal quantum dot systems, researchers are looking to create easier-to-produce solar panels with a greater efficiency than those currently available.

\section{Purpose of the research reported in this Thesis}

The purpose for the research reported in this thesis is to identify quantitative differences of colloidal systems examined under normal conditions and colloidal systems under microgravity aboard the International Space Station (ISS). By doing so, it seeks to improve the knowledge base for future nanoparticle haloing systems. This work continues research performed by Xiaoting Hong and Qingwen He on the colloidal science regarding nanoparticle haloing (NPH).

\section{Literature Review}

Nanoparticle haloing is the formation of a non-absorbing nanoparticle layer around small particles that induces an effective electrostatic repulsion between the microparticles. The concept of nanoparticle haloing was first published by researchers from the University of Illinois UrbanaChampaign and Carnegie Mellon University in 2001 (Valeria Tohver, 2001). The researchers showed that the charged nanoparticle species in solution migrated to regions surrounding uncharged microparticles. Tohver, used uniform silica spheres of radius $285 \mathrm{~nm}$ and zirconia nanoparticles with an average size of $3 \mathrm{~nm}$. The suspensions were prepared by adding low nanoparticle volume fractions $(0.05-0.45)$ to deionized water. Similar mixtures without 
nanoparticles were made for the purpose of zeta potential analysis. The nanoparticlemicroparticle mixtures were tested using zeta potential analysis, adsorption measurements and phase-behavior testing. Their work showed that zeta potential, phase behavior, and the quantity of nanoparticles adsorbed were all affected by nanoparticle concentration. In particular, their work showed that the phase of the colloidal system changed from a gel to a fluid in a narrow region of concentrations, as shown in Figure 1. The symbol $\phi$ represents the volume fraction of each component.

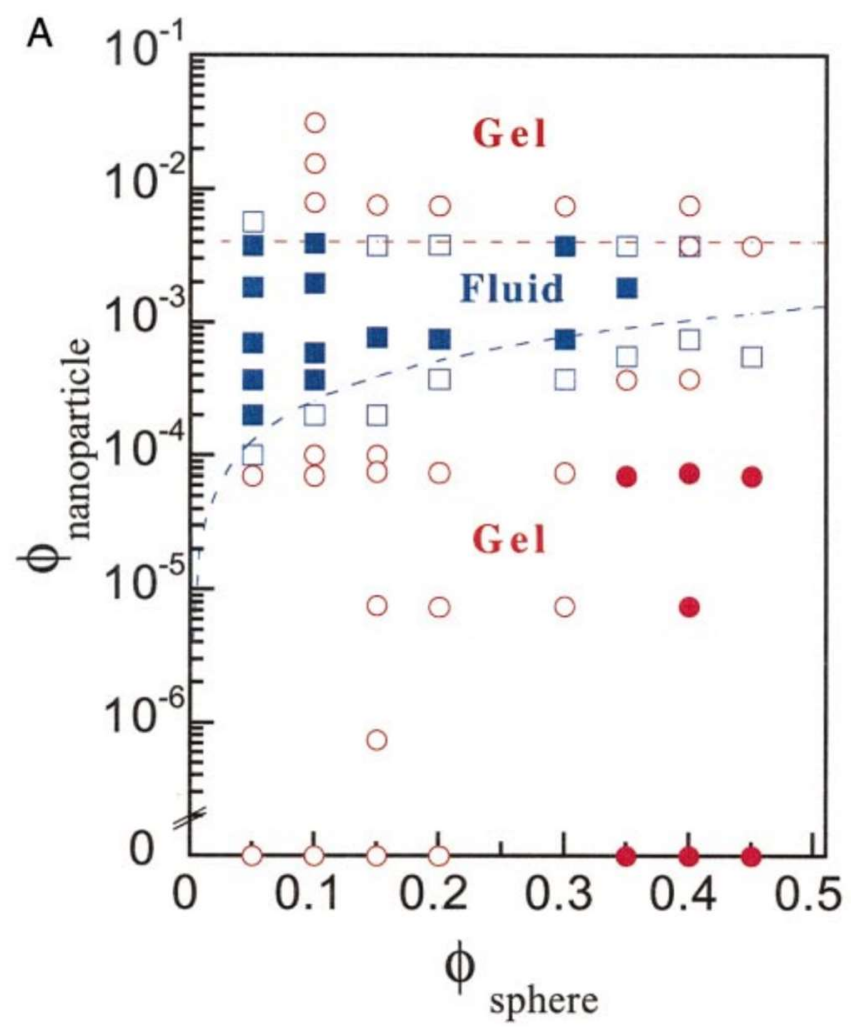

B

FIGURE 1: A PHASE DIAGRAM OF UNCHARGED MICROSPHERES AND REPULSIVE NANOPARTICLES (Valeria Tohver, 2001) 
Building on the work of Tohver, Everett Scheer and Kenneth Schweizer studied the equilibrium structure of bimodal colloidal mixtures (Everett N. Scheer, 2008). They observed the effect of size asymmetry between the nanoparticles and microparticles, as well as the effect of volume fraction and charge. The study confirmed the previous findings regarding gelation, as well as simulating the systems to compare lab and analytical data. The success of the researchers at Carnegie Mellon University as well as the lab headed by Scheer led to key questions regarding to the formation of nanoparticle haloing systems by researchers at the University of Louisville. How would the colloidal interaction forces vary as the particles neared one another? How would microparticlenanoparticle particle suspensions differ when exposed to microgravity? The first question was explored in the work of work of Xiaoting Hong (Hong, 2009). The second provides the basis for this study.

\section{E. Theory}

Colloids as a collection of particles are governed by several forces on the system. These include attractive Van der Waals forces and electrostatic double-layer forces. To achieve a thermodynamically stable system, the forces involved must be balanced. This primarily involves balancing the Van der Waals forces with a suitably repulsive stabilization force (L. A. Hough, 2013).

Van der Waals forces are primarily concerned with intermolecular phenomena; however, they have a measurable effect on larger colloidal particles as well. Van der Waals forces are comprised of three separate interactions, including London dispersion forces, dipole-dipole interactions, and dipole-induced dipole interactions (Lifshitz, 1992). 
The London dispersion force results when the electrons in two adjacent atoms occupy positions that make them temporary dipoles. The dispersion force relies heavily on the temporary distribution of electrons, and thus remains the weakest of the Van der Waal forces.

Dipole-dipole interactions occur when two molecular dipoles near each other in physical space. A dipole is a molecule that has an unequal sharing of electrons between atoms. This occurs due to the variance in atomic electronegativity and molecular geometry. When this occurs, electrons are drawn towards one region of the molecule, causing it to become more electronegative. When these dipoles interact, the partial positively charged end of one dipole attracts the partial negatively charged end of another dipole, causing a net attractive force.

Dipole-induced dipole interactions occur between a dipole and a molecule of evenly distributed electronegativity. When a dipole nears an atom or molecule with no dipole movement, it induces an electronic asymmetry in the other molecule, causing it to develop its own instantaneous dipole moment. When this occurs, the opposing dipole poles create an attractive force.

The Van der Waals forces affect both molecules and larger units, including colloids (Israelachvili, 2015). The expression for attractive forces between two macroscopic bodies based on London forces was derived in 1937 by Hugo Hamaker (Hamaker, 1937). Hamaker derived the expression using the assumption that the particles behaved like two isolated molecules in a vacuum. The Hamaker constant $(A)$ can be calculated for a Van der Waals body-body interaction as follows.

$$
A=\pi^{2} \times C \times \rho_{1} \times \rho_{2}
$$


In this instance $\rho_{1}$ and $\rho_{2}$ are the number densities of the two particles and $C$ is the particleparticle pair interaction coefficient. The Hamaker constant can then be used to calculate the interaction parameter $C$ from the potential between the two bodies where $r$ is the particle radius and $w(r)$ is the potential between the bodies.

$$
w(r)=-C / r^{6}
$$

When the colloidal sizes are much larger than the distance between them, a simplified expression applies:

$$
F_{v d w}(D)=-\frac{A R}{6 D^{2}}
$$

Where $A$ is the Hamaker constant $\left(0.8 \times 10^{-20} \mathrm{~J}\right)$ and $R$ is the radius of the microparticle.

Electrostatic (charge) stabilization can vary greatly depending on the surface potential and charge density of the particles, as well as the properties of the fluid medium the particles are suspended in. The charge of the particles depends on the particle material as well as charge density of the particles. If two particles are of the same charge and charge density, the result will likely be a repulsive effect. When particles are of heterogeneous charge, opposing charges may align to create an attractive force. Finally, if the charges are both positive but have differing magnitudes, the force may still be attractive due to a phenomenon known as the double-layer effect.

For this experiment the microparticles were comprised of silsesquioxane $\left(\mathrm{SiO}_{1.5}\right)$ which is itself not significantly electrostatically repulsive under the conditions of interest. For other 
colloidal systems however the particle charge is chosen so that it sufficiently counteracts the attractive Van Der Waals forces.

In silica particles, adsorption of ions to the particle surface charges a previously uncharged particle. Surrounding the particle surface is a secondary layer of oppositely charged ions, which drops off exponentially until it transitions to the bulk fluid. Between the adsorbed ions and the secondary counter-ions resides the Stern layer, which differentiates the two. Finally, the slipping plane differentiates between the secondary counter ions and the bulk fluid. Figure 2 provides an illustration of the double-layer system once it has fully developed in an aqueous charged solution.

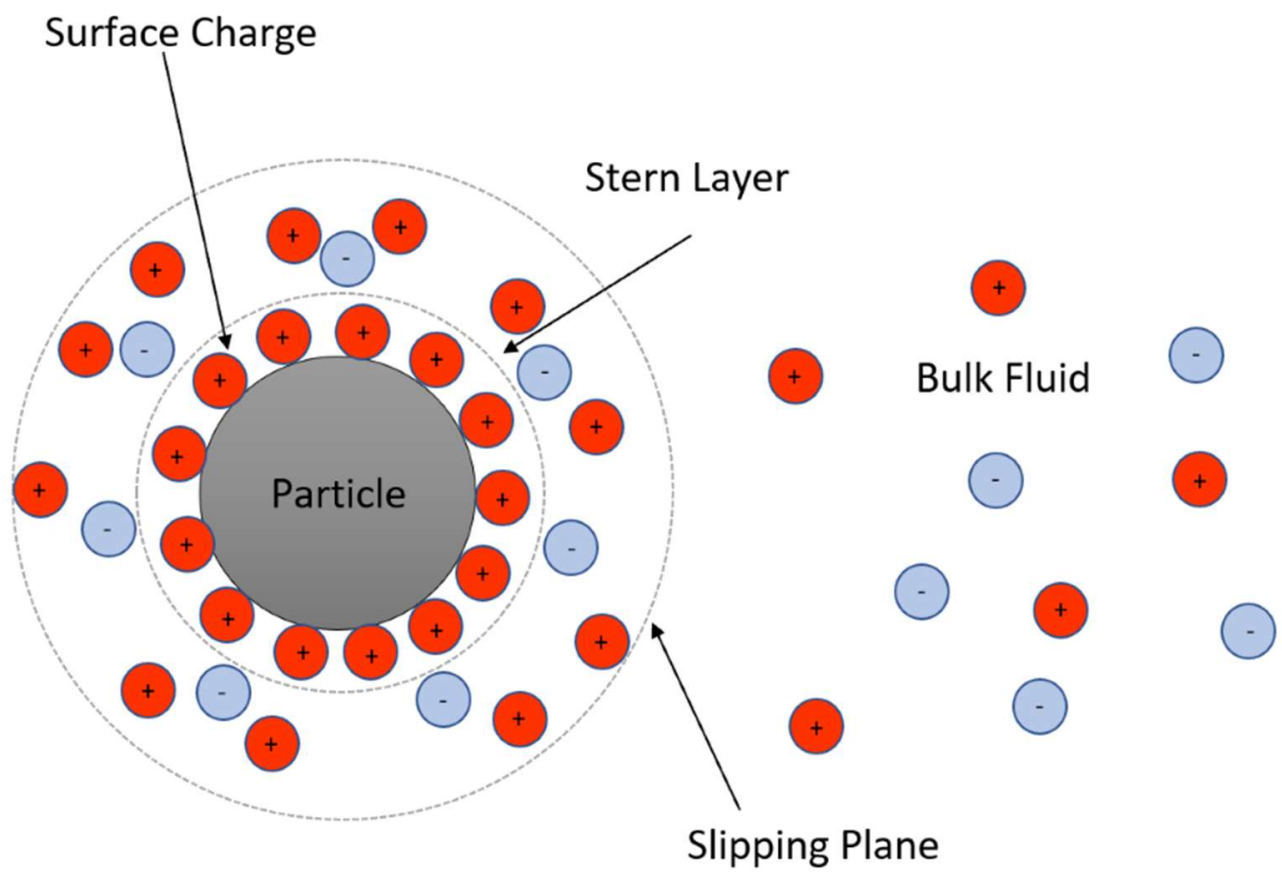

FIGURE 2: AN ILLUSTRATION OF THE DOUBLE-LAYER EFFECT WITHIN A CHARGED PARTICLE SYSTEM 
The slipping plane (also called the shear plane) is defined as the plane where the liquid velocity relative to the velocity of the particle is zero. If the liquid has a low dielectric constant and no ions adsorb to the particle, then the slipping plane may exist close to the particle surface. The Debye length is a measure of the distance a charge carrier's electrostatic effect persists in a colloidal system.

For the systems studied here, however, the double-layer effect does occur, and the slipping plane occurs at the boundary between the counter ions and the bulk fluid (Ross, 1988). The Zeta potential is defined as the potential difference between the stationary plane attached to the particle and the bulk fluid, or the potential difference across the slipping plane (Hunter, 1981). The Zeta potential can affect the stability of the resultant colloid, as shown in Table 2.

TABLE II: ZETA POTENTIAL VS. STABILITY BEHAVIOR (Kumar, 2017)

\begin{tabular}{|c|c|}
\hline Zeta Potential $(\mathrm{mV})$ & Stability behavior \\
\hline 0 to \pm 5 & Flocculation or Coagulation \\
\hline \pm 10 to \pm 30 & Incipient instability \\
\hline \pm 30 to \pm 40 & Moderate stability \\
\hline \pm 40 to \pm 60 & Good stability \\
\hline Greater than \pm 60 & Excellent stability \\
\hline
\end{tabular}


For both the ground nanoparticle tests and the microgravity tests, the Zeta potential of the silsesquioxane particles was kept at 0 to $+5 \mathrm{mV}$. This was done to isolate the nanoparticle repulsion from standard double-layer repulsion. An example of electrostatic repulsion is illustrated in Figure 3a.

a)
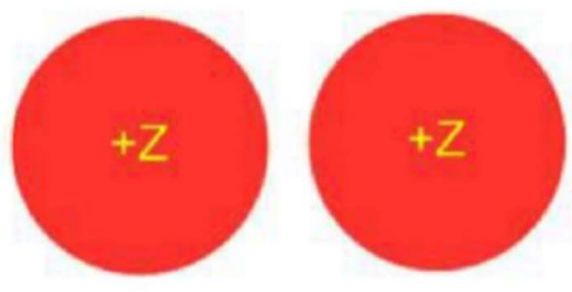

b)
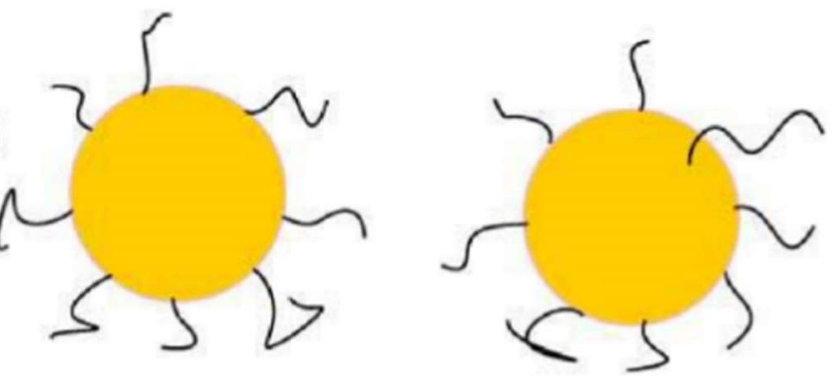

c)

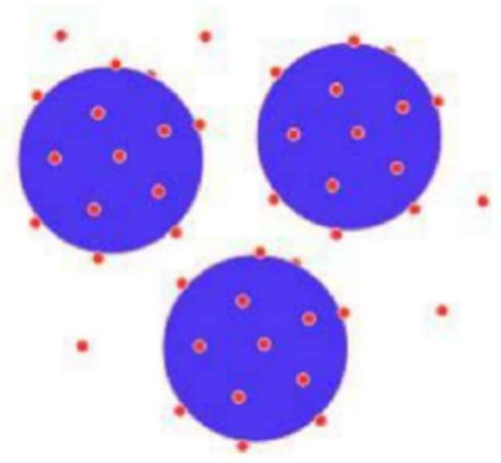

FIGURE 3: COLLOIDAL STABILIZATION MECHANISMS INCLUDING a) ELECTROSTATIC b) STERIC AND c) NANOPARTICLE HALOING

Steric hindrance can also be utilized to provide colloidal stabilization. To provide steric hindrance, polymer chains are adsorbed to the surface of the particle. The adsorbed polymers 
extend off the particle like hairs, as shown in Figure 3b. The steric repulsion acts via two effects; volume restriction and osmotic diffusion (Napper, 1976). When two such particles interact, their polymeric chains interact. The proximity of chains to each other decreases the particles entropic free energy and makes the particle proximity a less-preferred state. Additionally, the interaction of the particles causes the polymer chains to increase in concentration. This creates an osmotic repulsion between the water and the polymers. When the particles separate, the concentration lessens, and the osmotic repulsion reduces.

Finally, nanoparticle haloing is used as a stabilization mechanism within colloidal systems. Nanoparticle haloing involves a bimodal system using negligibly charged microparticles with charged nanoparticles. At specific concentrations the nanoparticles are observed to maintain a nonzero separation distance between them and the microparticles. In 2001, this novel method was discovered by Tohver et al (2001). They observed that uncharged silica microparticles can be suspended by adding small amounts of charged zirconia nanoparticles. In 2008 Zhang et al. utilized ultra small-angle X-ray scattering to quantify the nanoparticle-microparticle separation distance. At a nanoparticle volume fraction of $10^{-3}$, the nanoparticle layer was measured to reside $2 \mathrm{~nm}$ away from the colloidal surface at a $\mathrm{pH}$ of 1.5 . This separation distance is approximately equal to the Debye Length.

In 2014 Qingwen He performed direct force measurements of a nanoparticle haloing system using an atomic force microscopy (AFM) (He, 2014). This was a continuation of work done by Hong in 2009 (Hong, 2009). AFM uses a light lever to detect the deflection of a cantilever as it interacts with the substance below it. A laser is focused on the cantilever spring, which reflects the light onto a split photodiode detector as shown in Figure 4. The amount of 
deflection can be accurately gauged based on the output of the photodiode. The force experienced by the cantilever can be calculated using Hooke's Law.

$$
F_{s}=k x
$$

The cantilever head can move in the $x, y$, and $z$ axis to map out the surface and force applied. In this test, the cantilever moved vertically to measure the force on a single $1 \mu \mathrm{m}$ silica particle suspended in a nanoparticle haloing solution.

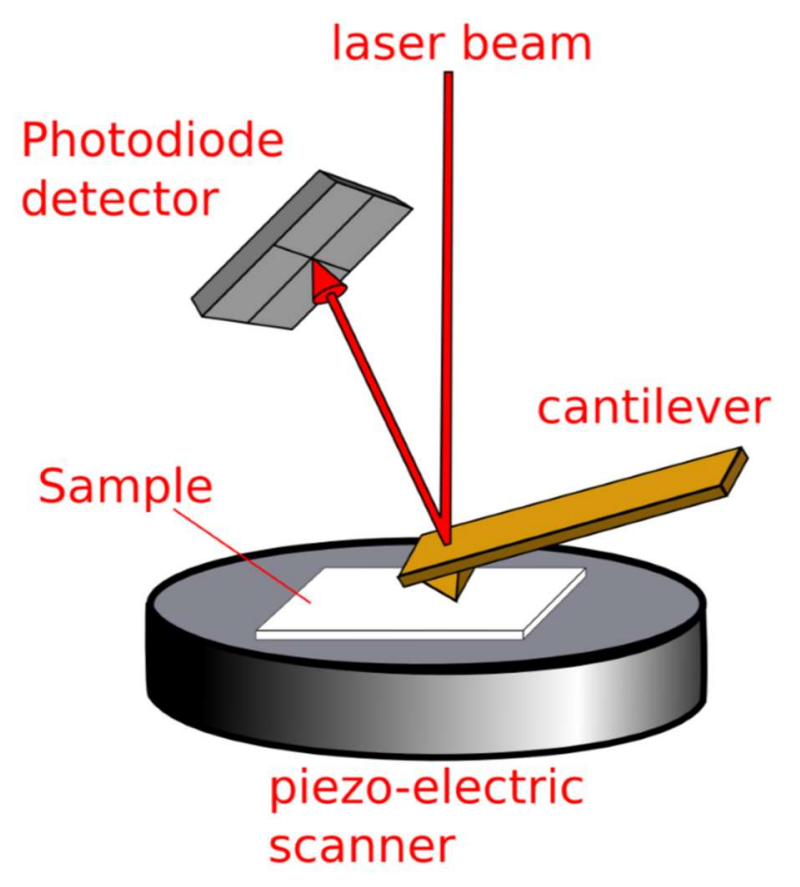

FIGURE 4: THE DESIGN OF AN ATOMIC FORCE MICROSCOPE (He, 2014)

The experiment tested two haloing configurations: the force between a single silica particle and a silica plate suspended in nanoparticles. The study showed that the electrostatic repulsion of the nanoparticle haloing system increases as the nanoparticle concentration increases, as shown in Figure 5. The study confirmed a repulsive force that occurred due to nanoparticle haloing. It found 
that a suitable haloing condition could be met with nanoparticle volume fractions between $10^{-3}$ and $10^{-5}$.

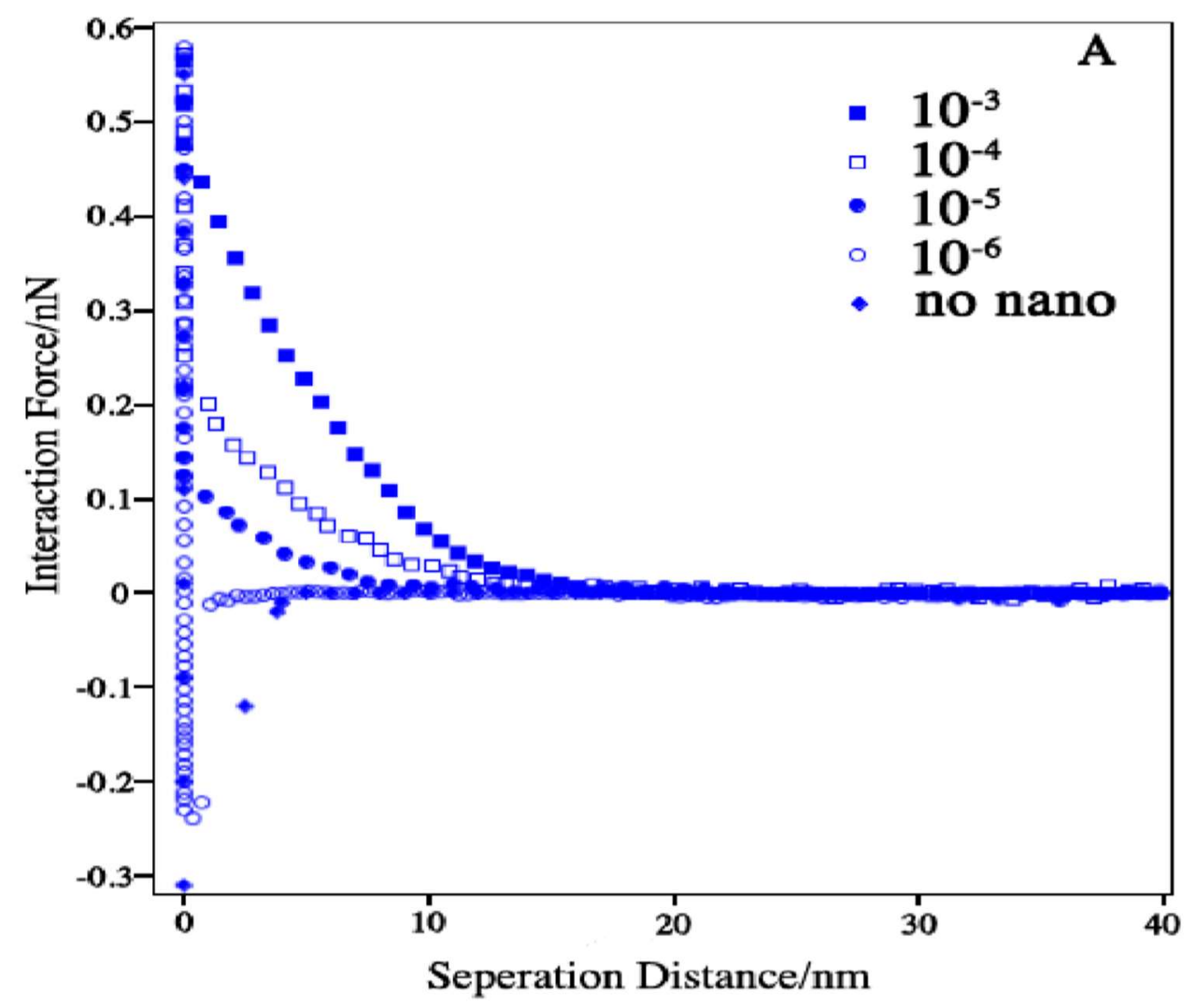

FIGURE 5: INTERACTION FORCE ON A SILICA MICROPARTICLE IN A NANOPARTICLE HALOING SYSTEM (He, 2014)

The study by He performed three important tasks. First, she directly measured the force applied on a microparticle due to nanoparticle haloing at multiple separation distances. Secondly, it confirmed the findings made by previous studies. Third, she demonstrated the impact of the depletion interaction even at small nanoparticle volume fractions. Based on the previous nanoparticle haloing systems developed by Tohver, He, and others, a procedure was developed to test the nanoparticle haloing system when in microgravity conditions. 


\section{$\underline{\text { The International Space Station }}$}

The International Space Station (ISS) is an international project whose construction began in 1998 and finished in 2010. Its structure is modular, containing modules for control, viewing, research, and even exercise. The ISS spans the area of a football field and weighs over 800,000 lbs. (Howell, 2018). It is aided by mission control centers in both Houston and Moscow and is inhabited by to 6 astronauts and cosmonauts at a time. While there, its inhabitants conduct scientific research such as testing the long-term health effects of spaceflight. Research also includes testing commercial 3D printers and conducting biological experiments on rats and plants. Included in the ISS is the U.S. Destiny Laboratory, a multi-purpose research module which launched aboard the space shuttle in 2001. This module contains the Fluids Integrated Rack (FIR) which provides data, power, and temperature management to the instruments inside. Included in these instruments is the Light Microscopy Module (LMM) which conducts imaging experiments in microgravity conditions.

\section{The Light Microscopy Module}

The Light Microscopy Module is a fully automated laboratory microscope (Leica RXA). Its purpose is to perform biological and physics-based experiments without the need of monitoring by the astronauts. The LMM can accept sample cells, modules that contain the experiment samples inside of a functional container. These sample cells include the Constrained Vapor (CVB) module which studies heat transfer under phase change, the LMM Petri base 
which can contain biological experiments, and the ACE-T module which studies colloidal systems (Reckart, 2019). The experiments conducted for this research utilized the ACE-T module. The ACE-T module contains both mixing and heating capabilities, allowing samples to be mixed in-situ. The experiments made by the LMM study the effect of microgravity on samples such as crystal growth and cellular behavior.
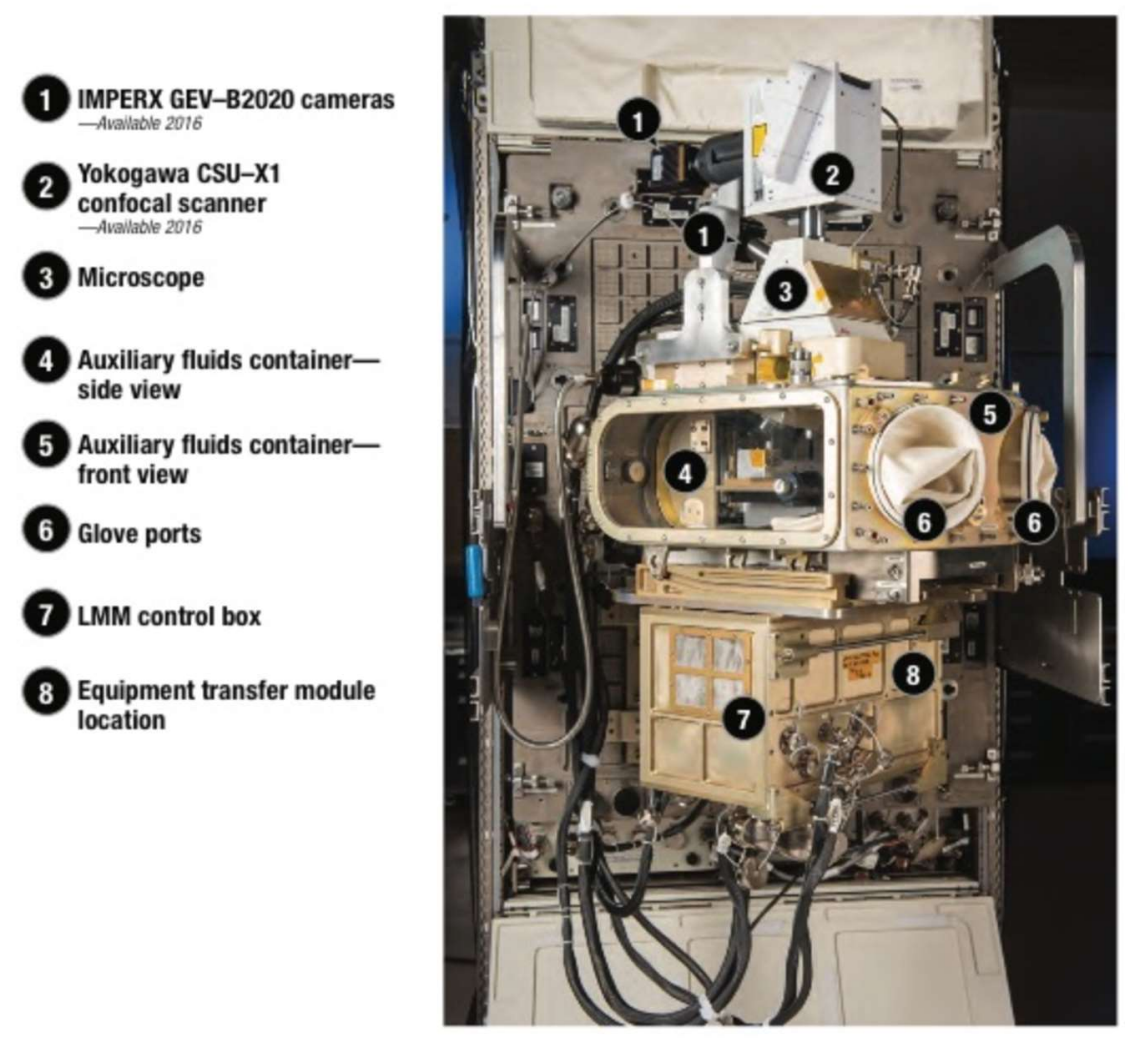

FIGURE 6: COMPONENTS OF THE LIGHT MICROSCOPY MODULE (Reckart, 2019) 


\section{METHODS}

\section{$\underline{\text { Overview }}$}

The bimodal colloidal system of particles was made from microparticles and nanoparticles suspended in a nitric acid solution. The nanoparticles were sourced from Nyacol Nano Technologies in an aqueous sodium acetate solution of $4.37 \mathrm{vol} \% \mathrm{ZrO}_{2}$ nanoparticles $(\mathrm{Zr} 10 / 20)$. The nanoparticles ranged from $6 \mathrm{~nm}$ in diameter to $20 \mathrm{~nm}$ in diameter with an average diameter of $10 \mathrm{~nm}$. The silsesquioxane microparticles were synthesized from raw materials and functionalized with Rhodamine B using a previously published technique discussed later in this section (Niharika Neerudu, 2017). The 1\% microparticle solution in nitric acid was tested for zeta potential in order to determine the isoelectric point for the system using a 90Plus Particle Size Analyzer. The isoelectric point was determined to be at a $\mathrm{pH}$ of 2.80, which was used in the development of future

systems. The $4.37 \mathrm{vol} \% \mathrm{ZrO}_{2}$ suspension was diluted to a $1.00 \%$ by volume suspension. Final 
suspensions were then prepared by mixing the microparticles in the nitric acid and adding the $1.00 \%$ nanoparticle solution. The mixture was sonicated and loaded into capillary tubes, then capped with wax Each capillary tube was placed into a 3-d printed capillary holder and loaded into a Nikon Eclipse Ti microscope. The microscope captured 8-bit confocal images $15 \mu \mathrm{m}$ into the sample at a rate of 4 images/minute.

\section{$\underline{\text { Silsesquioxane Microparticle Synthesis }}$}

Silsesquioxane is an organosilicon compound with the molecular formula $\left[\mathrm{RSiO}_{1.5}\right]_{\mathrm{n}}$ where $\mathrm{R}$ is an external group attached to the main structure, as shown in Figure 7 below. Each silicon atom is attached to three oxygen groups and an $\mathrm{R}$ group. These available bonds allow the Silsesquioxane to be functionalized with groups such as dyes.

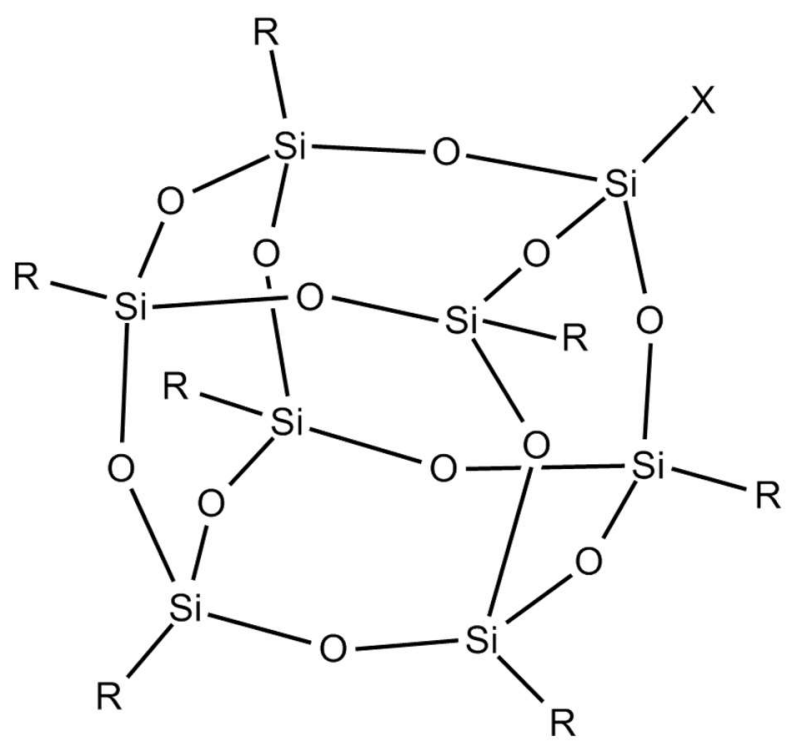

FIGURE 7: A REPRESENTATIVE UNIT STRUCTURE OF SILSESQUIOXANE

Benzyl Chloride Silsesquioxane (BC-SSQ) particles were prepared utilizing a modified Stöber method (Neerudu, 2017). In this method, p(chloromethyl)phenyltrimethoxy silane is 
catalyzed with ammonium hydroxide in a hydrolysis reaction. The synthesis process is shown in Figure 8 below.

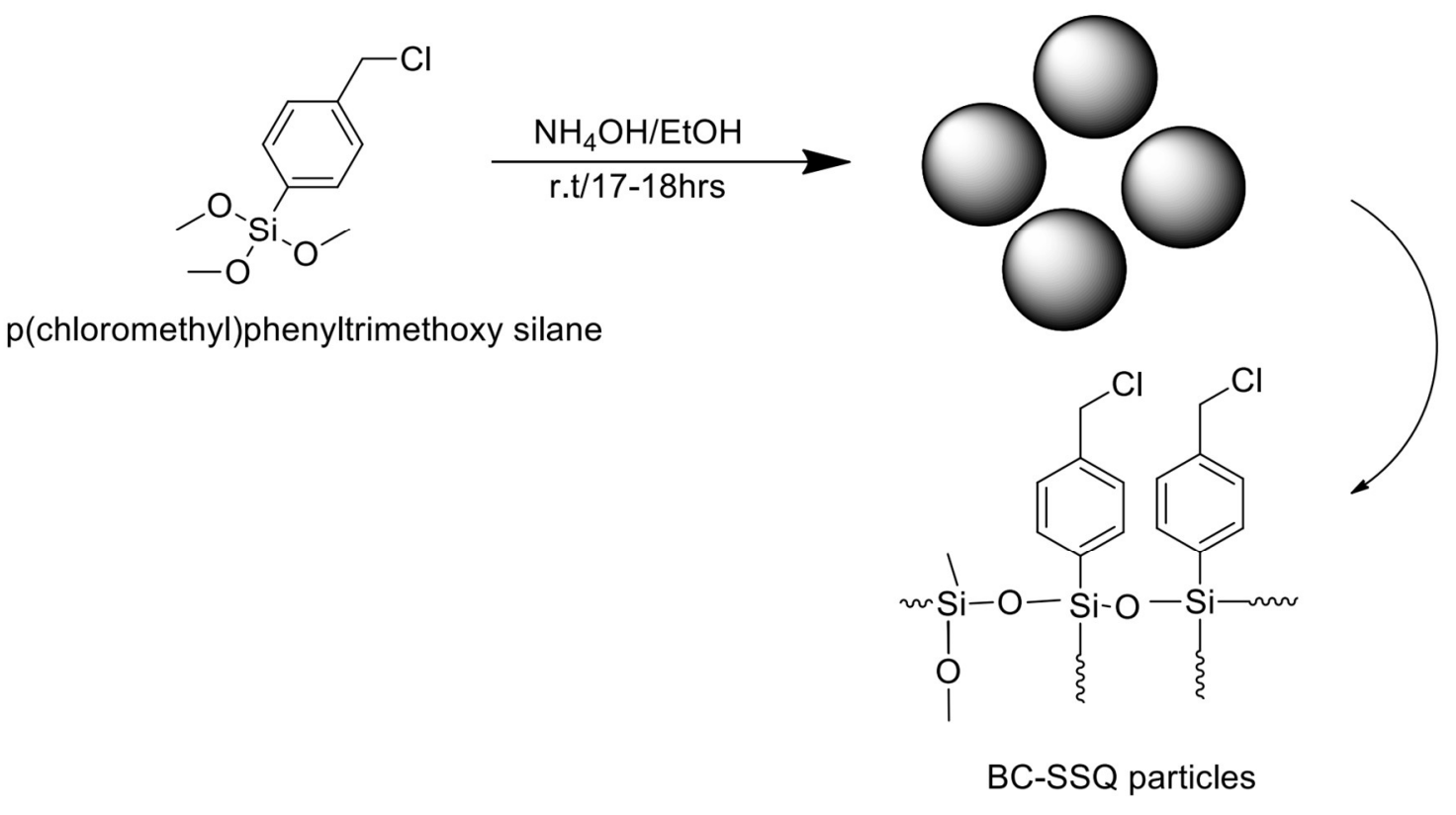

FIGURE 8: SYNTHESIS OF BENZYL CHLORIDE SILSESQUIOXANE MICROPARTICLES (Sreeramulu, 2016)

The following synthesis was performed in the fume hood under safe laboratory conditions. $10 \mathrm{~mL}$ of anhydrous ethanol and $1 \mathrm{~mL}$ of ammonium hydroxide were added to a $20 \mathrm{~mL}$ vial. The vial contents were stirred for five minutes. Benzyl chloride trimethoxy silane was added at a rate of $0.25 \mathrm{~mL}$ per minute until $1.8 \mathrm{~mL}$ was added. The mixture was stirred overnight at room temperature. The particles were separated from the mixture by centrifuging at $3000 \mathrm{rpm}$ for twenty minutes. Particles were washed six times with alternating ethanol and water and centrifuging between each wash. The resultant particles were a dry, white powder. 
For this researched, imaging was performed using confocal microscopy. Confocal microscopy uses a focused laser to image a single plane of the sample at a time. For this reason, particles were needed that fluoresced under a $532 \mathrm{~nm}$ wavelength light. Rhodamine B was chosen for this experiment as a dye that fluoresced in the correct wavelength of light and could be attached to the Silsesquioxane microparticles. The functionalization process is shown in Figure 9 below.

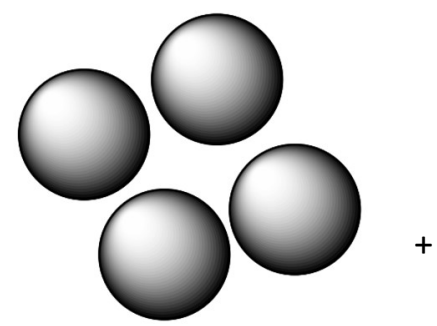

BC-amine-SSQ particles

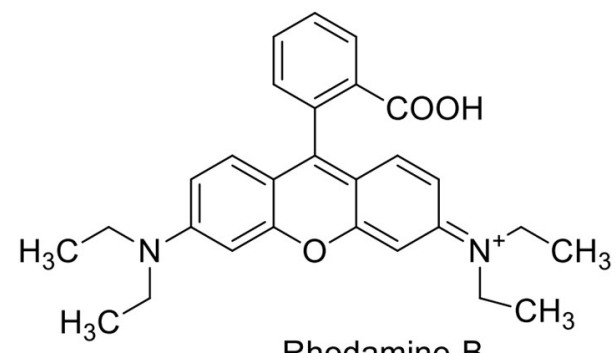

Rhodamine-B

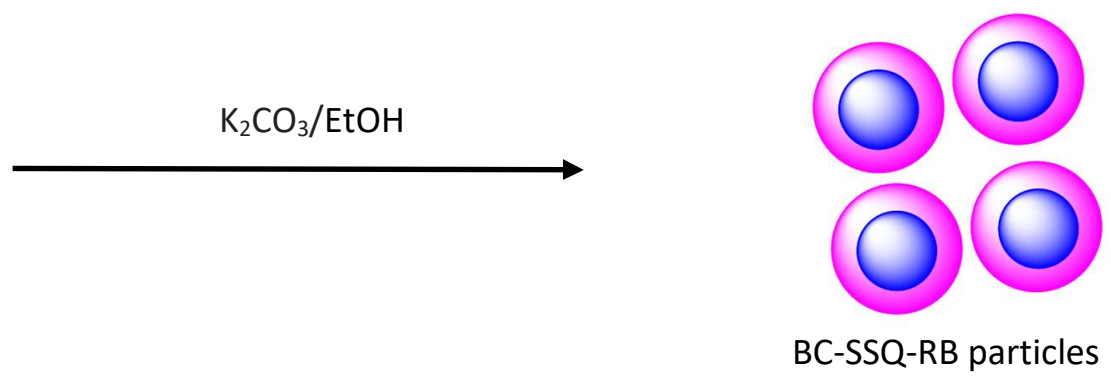

FIGURE 9: FUNCTIONALIZATION OF BC-SSQ WITH RHODAMINE B (Sreeramulu, 2016)

The microparticles were functionalized using the following procedure. $500 \mathrm{mg}$ of BC-SSQ particles and $30 \mathrm{~mL}$ anhydrous ethanol were added to a $100 \mathrm{~mL}$ round-bottom flask. The mixture was stirred for five minutes until the particles were completely dispersed. $79 \mathrm{mg}$ of potassium carbonate was added to the flask and stirred until fully mixed. Finally, $100 \mathrm{mg}$ of Texas Red rhodamine B was added and mixed overnight. The mixture was separated via centrifugation at $3000 \mathrm{rpm}$ for twenty minutes and then washed twelve times using alternating solutions of water and ethanol. For each wash, the solvent was added and mixed well via a Vortex Genie 2 vortexing 
machine. Each sample was then centrifuged for 20 minutes at $3000 \mathrm{rpm}$. The resultant particles were a bright pink hue and fluoresced under a $532 \mathrm{~nm}$ light.

\section{Zeta Potential Analysis}

Zeta potential analysis was used to determine the $\mathrm{pH}$ of the isoelectric point. Suspensions of microparticles in nitric acid were prepared as follows. Benzyl chloride Silsesquioxane functionalized with Rhodamine B dye (BC-SSQ-RhB) was added $(0.66 \mathrm{mg})$ to $3.996 \mathrm{~mL}$ of nitric acid. Nitric acid solutions of a specific $\mathrm{pH}$ were prepared by mixing a $\mathrm{pH} 1 \mathrm{HNO}_{3}$ solution with de-ionized water. The solutions were allowed to mix overnight before sample preparation. The mixture was first vortexed and then sonicated using a sonicator (Qsonica Q500) for five minutes. The $4 \mathrm{~mL}$ samples were then tested for zeta potential using a 90Plus Particle Size Analyzer (Brookhaven Instruments, Brookhaven, NY). Each sample used was tested six times to ensure numerical accuracy. Between each test, the sample was remixed well using a volumetric pipet. Based on the results, the isoelectric point of the microparticles was determined to occur at a $\mathrm{pH}$ of 2.80. For future samples, nitric acid of $2.80 \mathrm{pH}$ was used as the background solution.

\section{Sample Preparation}

Zirconia nanoparticles were first sourced from Nyacol Nano Technologies Inc. as a $20 \mathrm{wt}$. $\%$ aqueous suspension. The suspension was then diluted to $1 \%$ by vol in a $10 \mathrm{~mL}$ flask by adding $0.225 \mathrm{~mL}$ of $\mathrm{ZrO}_{2}$ solution to $9.775 \mathrm{~mL}$ of $2.8 \mathrm{pH} \mathrm{HNO}_{3}$. This suspension was then sonicated for 5 minutes using a Qsonica Q500 sonicator and formed the base for future samples.

Samples were prepared in the following manner. The dyed microparticles were mixed into the system by adding $0.066 \mathrm{~g}$ to the appropriate amount of nitric acid, as shown in Table 3 below. 
To this mixture the 1 vol \% suspension of nanoparticles was added, and the mixture was then vortexed and sonicated for five minutes.

TABLE III: MIXING AMOUNTS FOR THE BIMODAL COLLOIDAL MIXTURE

\begin{tabular}{|c|c|c|c|c|c|}
\hline MP Vol\% & NP Vol \% & Total Volume $(\mathrm{ml})$ & MP (g) & NP (ml) & $\begin{array}{c}\text { HNO3 } \\
(\mathrm{ml})\end{array}$ \\
\hline $1.00 \%$ & $0.01 \%$ & 4 & 0.06600 & 0.040 & 3.92 \\
\hline $1.00 \%$ & $0.055 \%$ & 4 & 0.06600 & 0.220 & 3.74 \\
\hline $1.00 \%$ & $0.10 \%$ & 4 & 0.06600 & 0.400 & 3.56 \\
\hline
\end{tabular}

\section{Ground Testing}

Seven total samples were prepared for imaging. The following description outlines the sample preparation process. The samples were imaged in borosilicate capillary tubes (Rectangle Boro Capillary Tubes from Vitrotube) with dimensions of $0.20 \times 2.00 \times 50.0 \mathrm{~mm}$. First, the $4 \mathrm{~mL}$ colloidal mixture was sonicated for five minutes to ensure particle separation and dispersion. Then, the mixture was transferred into the capillary tube and the ends of the tube sealed with capillary wax. 


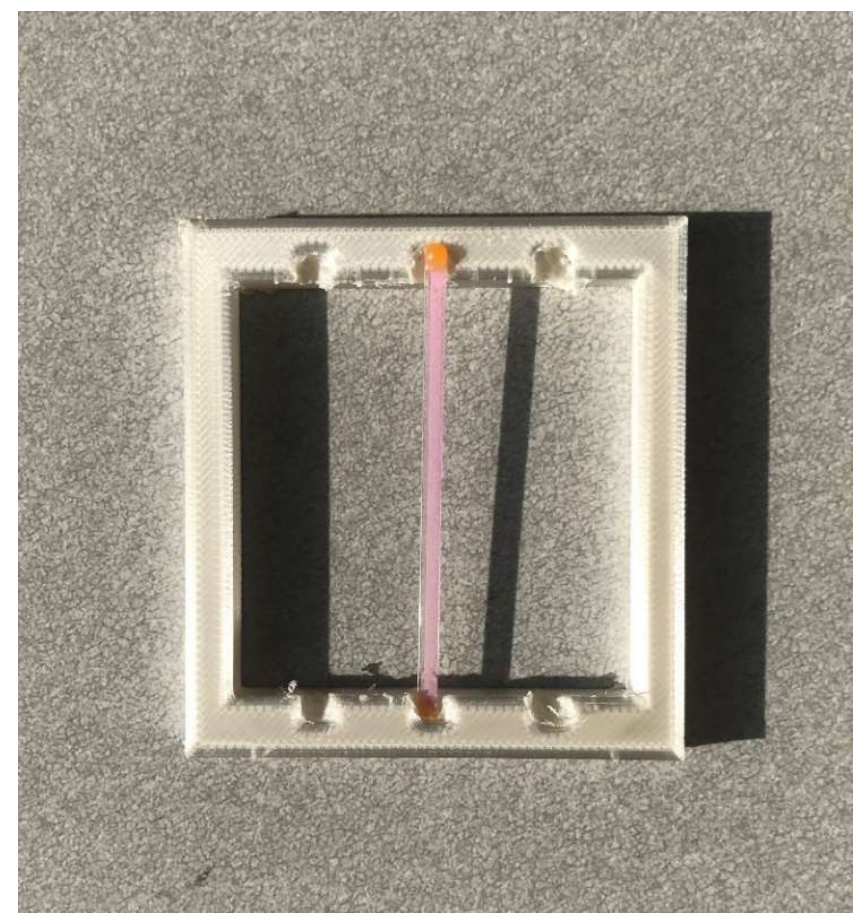

FIGURE 10: A SAMPLE CAPILLARY TUBE WITHIN THE 3D-PRINTED HOLDER

The capillary tube was transferred to a 3D-printed tube holder and loaded into a Nikon Eclipse Ti confocal microscope. The microscope is shown in Figure 11 below. This microscope included an Xon Ultra Andor confocal and imaging system which illuminated and imaged the particles at a rate of 4 images/minute. The images used a 200-millisecond exposure and were 512 x 512 pixels each. 


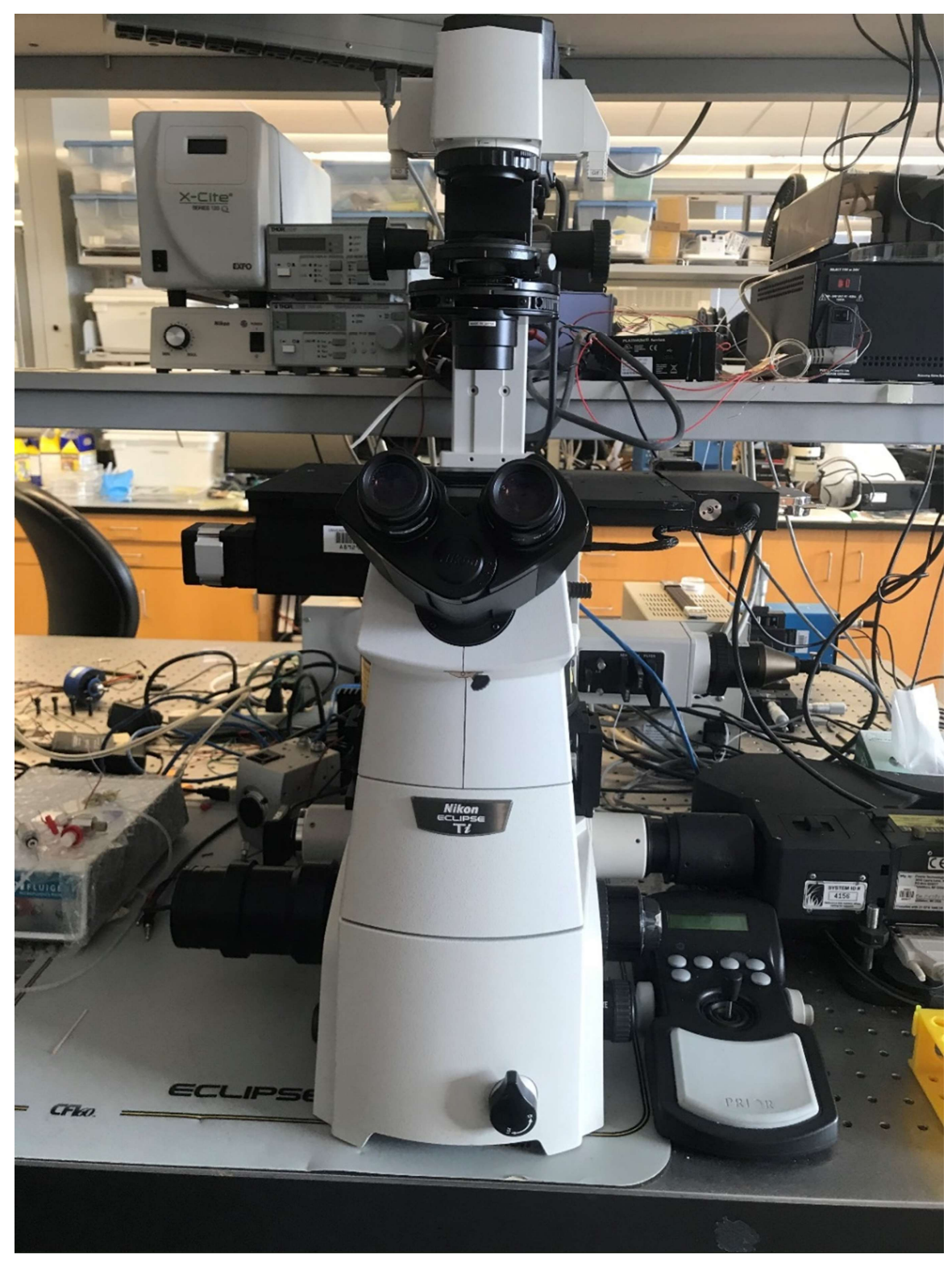

FIGURE 11: NIKON CONFOCAL MICROSCOPE FOR GROUND EXPERIMENTS

The focal depth was adjusted to be just above the bottom of the capillary tube $(\sim 15 \mu \mathrm{m}$ from the capillary wall) to ensure the smallest amount of light blockage possible. The microscope used was a Nikon Eclipse Ti, a modular system capable of both light field and confocal microscopy. A 20X lens was used with a $561 \mathrm{~nm}$ confocal laser to properly illuminate the particles. 
Images were taken every 15 seconds over a 1-hour period. This matched the chosen time frame of the microgravity testing.

\section{Microgravity Testing}

Microgravity samples were prepared in an identical fashion to the NPH ground samples. Samples of $0.01 \%, 0.055 \%$ and $0.1 \%$ by vol nanoparticles were mixed and sent to the Glenn Research Center in Cleveland, $\mathrm{OH}$ for pre-flight preparation. The samples were loaded into 3 borosilicate capillary tubes with the dimensions $0.20 \times 2.00 \times 50.0 \mathrm{~mm}$. Capillary 1 (C1) contained 0.01 vol\% nanoparticles, Capillary $2(\mathrm{C} 2)$ contained 0.055 vol\% nanoparticles, and Capillary 3 (C3) contained $0.1 \mathrm{vol} \%$ nanoparticles. A single micro-stirring rod (diameter $0.1 \mathrm{~mm}$ and $1 \mathrm{~mm}$ long) was loaded into each tube. The capillary tubes were capped with wax and a polymeric cap to prevent diffusion. The capillary tubes were loaded into an integrated imaging and mixing module, which was loaded into a SpaceX Falcon 9 Dragon capsule (CRS 16) and delivered to the International Space Station (ISS) on December $5^{\text {th }}, 2018$ for microgravity imaging. An image of the integrated module with the capillary tubes is shown below.

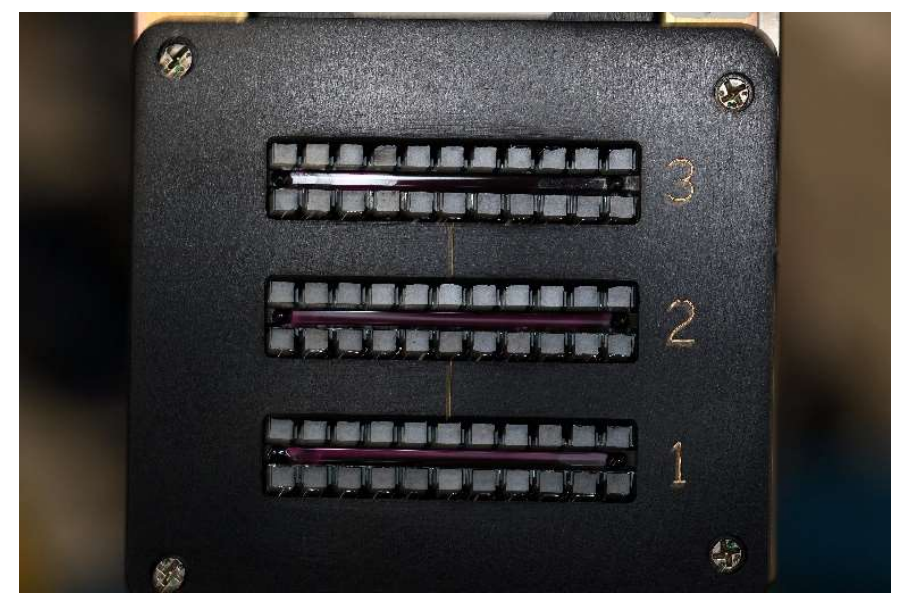

FIGURE 12: NPH SAMPLES LOADED INTO THE INTEGRATED IMAGING AND MIXING MODULE 
Testing began on May $20^{\text {th }}, 2019$. Each imaging sequence began with a series of $2.5 \mathrm{X}$ images. Before running an imaging test script, the confocal microscope used these low magnification images to determine the state of the capillary tubes. During this process the researcher present would select a region of interest (ROI) to focus on for the following test. The mixture would then be mixed by sending electromagnetic pulses to the stirring rod inside. Each capillary is housed within a set of inductors, as shown in Figure 12 above. These inductors produce a magnetic pulse that moves the stirring rod inside. The pulses both rotated the stirring rod in the mixture and translated it across the capillary tube.

This thesis focused on the $20 \mathrm{X}$ single-plane imaging tests. Once the mixing script concluded, the $2.5 \mathrm{X}$ preliminary script was used to determine a well-mixed ROI. When the ROI was selected, a 20X imaging script began. The confocal imaging system took images at 10 frames per second over the first 10 minutes, then slowed to 15 frames per minute for the remaining 50 minutes. This was to ensure that any fast-paced agglomeration that occurred during the first 10 minutes would be well documented. Select images were then sent down from the ISS over the medium-rate downlink (MRDL) to the Glenn Research Center. Once bandwidth was available, the full set of images was returned to earth using the high-rate downlink (HRDL). Five single plane settling scripts were successfully run for Capillary 2, the NPH mixture containing 0.055 vol \% nanoparticles. These images were then evaluated using blob size analysis.

\section{$\underline{\text { Blob Size Analysis }}$}

Blob size analysis is an evaluation technique used to determine the degree of particle agglomeration. The analysis was run using a macro in ImageJ. The user selected a region of 
analysis (ROI). The macro created a table to store the image data. The image was converted to 8 bit format, and a brightness threshold was set to differentiate the particles from the backdrop. A watershed function was used to mathematically separate blobs that were barely touching. The macro individually counted the blobs within the ROI and the blob area based on the results were then graphed and compared with the ground experiments, as discussed in the Results section. 


\section{RESULTS AND DISCUSSION}

\section{Zeta Potential Analysis and pH Adjustment Results}

Using the synthesis method described, twelve batches of silsesquioxane microparticles were synthesized. Each batch of silsesquioxane microparticles was characterized using zeta potential analysis. By making multiple batches, the zeta potential could be plotted both for differing $\mathrm{pH}$ values and across multiple batches. If a batch showed consistent zeta potential results, then the Rhodamine B fluorescent tag was added. Individual batches were judged based on their isoelectric point similarity to the rest of the batches, as well as the similarity between the functionalized and non-functionalized microparticles. For the ground experiments, Batch 12 was chosen for its zeta potential consistency. The results of the testing are shown in Table 4 below.

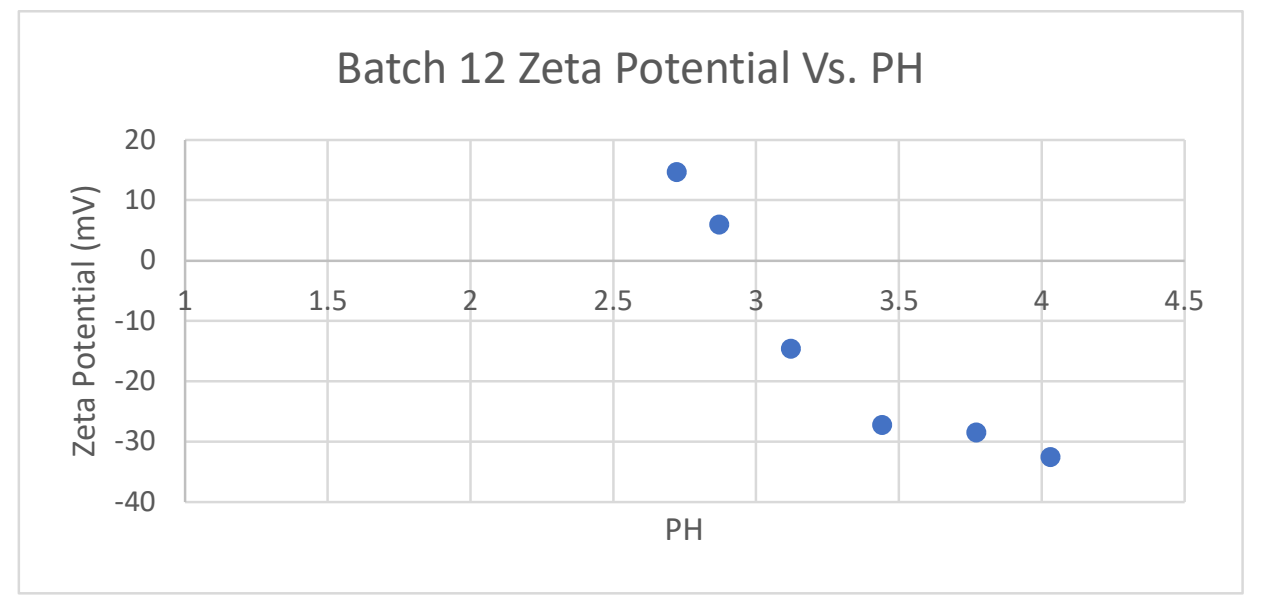

FIGURE 13: ZETA POTENTIAL RESULTS FOR BATCH 12 MP SAMPLES 
The results showed that the zeta potential increased as the $\mathrm{pH}$ decreased, with an isoelectric point at just under $\mathrm{pH} 3$.

Sample 12 was then functionalized with Rhodamine B, and the zeta potential tested again for varying $\mathrm{pH}$. The results showed consistency to the non-Rhodamine B sample, with a continuous curve and an isoelectric point close to $\mathrm{pH} 2.8$.

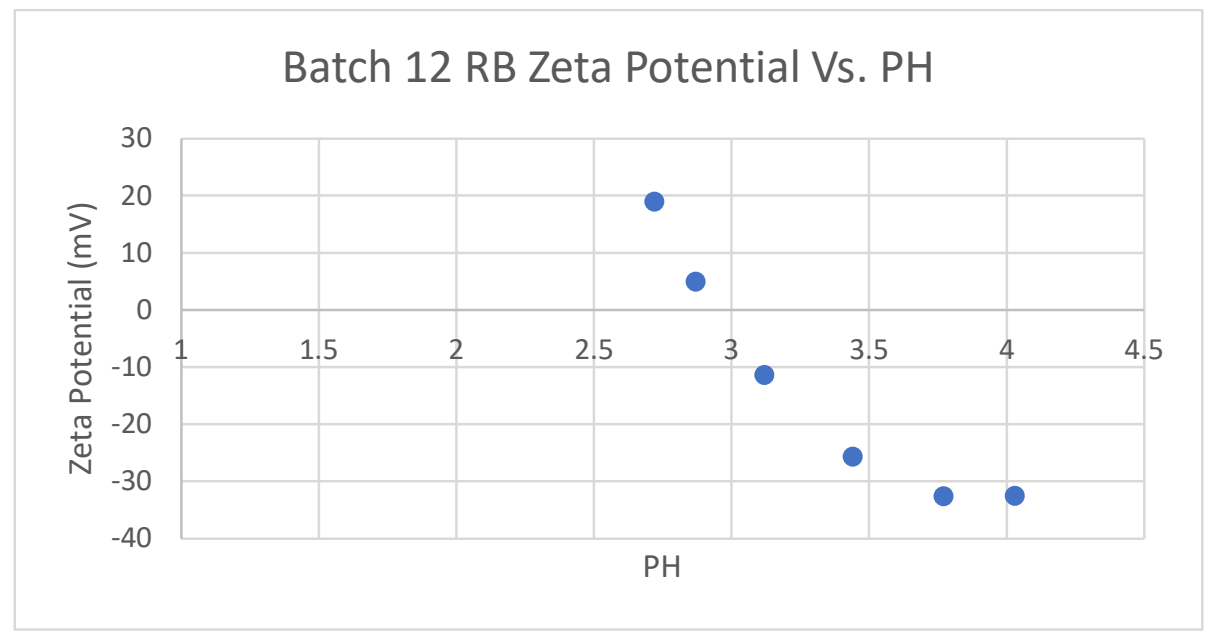

FIGURE 14: ZETA POTENTIAL RESULTS FOR BATCH 12 RB MP SAMPLES

Based on this data, batch 12 was chosen for ground analysis and the nanoparticle haloing solution mixed to be $2.80 \mathrm{pH}$. For the rest of the $\mathrm{pH}$ testing results, see APPENDIX I.

\section{$\underline{\text { Single-Plane imaging for Ground Experiments }}$}

The single-plane imaging was carried out over the course of a 2 -week period. At least 4 nanoparticle haloing mixtures were prepared for $0.1 \mathrm{vol} \%, 0.055 \mathrm{vol} \%$, and $0.01 \mathrm{vol} \%$ configurations. Each sample was sonicated and allowed to settle for 1 hour while images were taken using 20X confocal microscopy. Images were recorded every 15 seconds. Blob size analysis was run on the samples, and the results are shown below. The program reported average blob area in microns squared for each image. The blob size results were plotted over time, along 
with a running average over the forward 10 images. Run 2 for the $0.1 \%$ nanoparticle concentration is shown in Figure 15 below. For the entire set of ground haloing runs, see APPENDIX II.

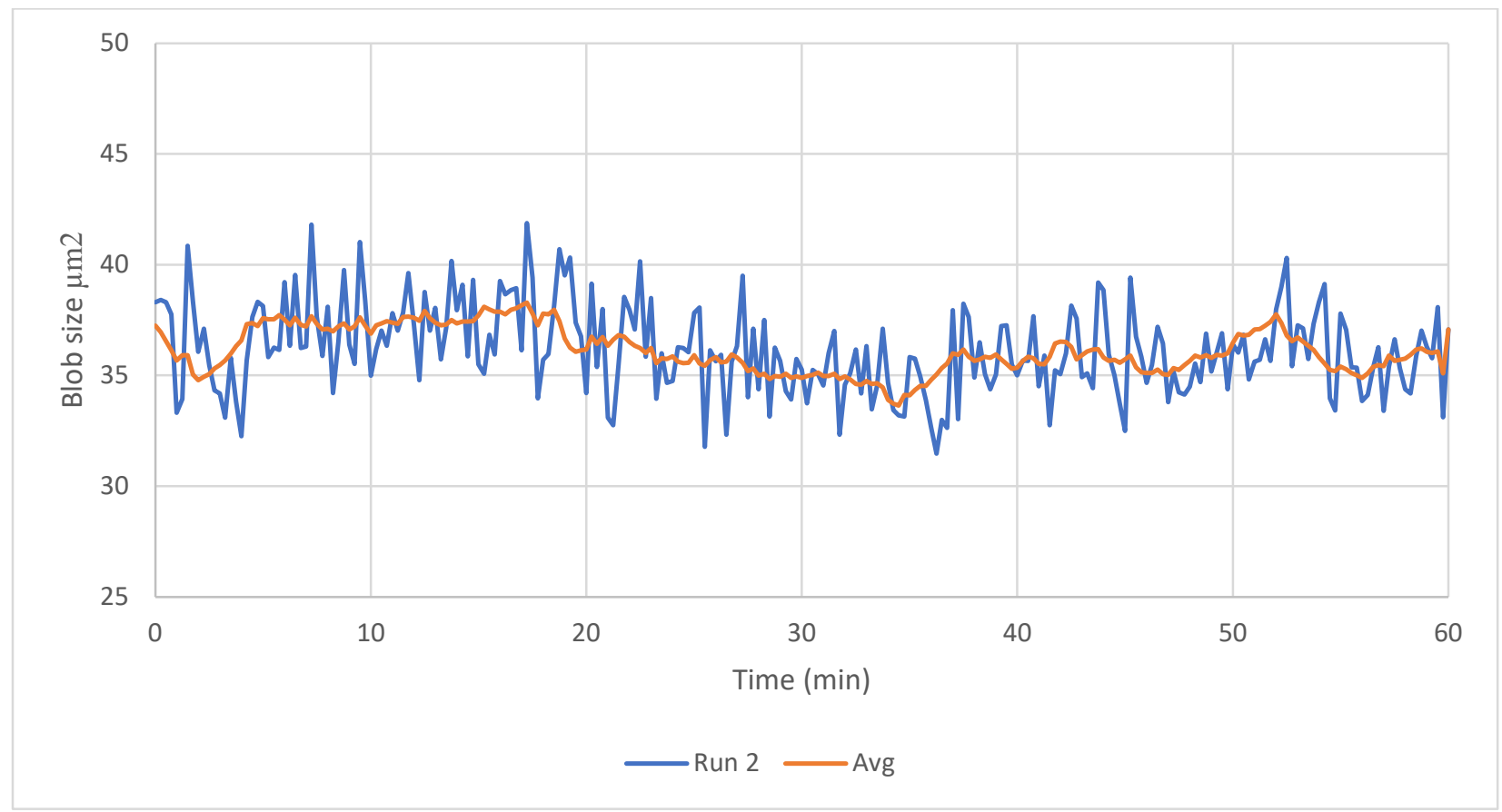

FIGURE 15: BLOB SIZE RESULTS FOR 0.1\% NANOPARTICLE RUN 2

Figure 15 shows the colloidal behavior for the 1-hour period through the lens of blob size. Due to variability in the program, a running average was used (shown in orange). This behavior was mimicked by Runs 4, 5, and 7, which either showed a gradual decrease or constant blob sizes. Run 1 showed a decrease in blob size that lasted until minute 20, when it settled to a constant blob size of roughly $30 \mu \mathrm{m}^{2}$. The decrease occurred from $37 \mu \mathrm{m}^{2}$ to $31 \mu \mathrm{m}^{2}$, which represented a change equal to the largest spike due to error in the analysis program $\left(6 \mu \mathrm{m}^{2}\right)$. Thus, this change was not significant. 


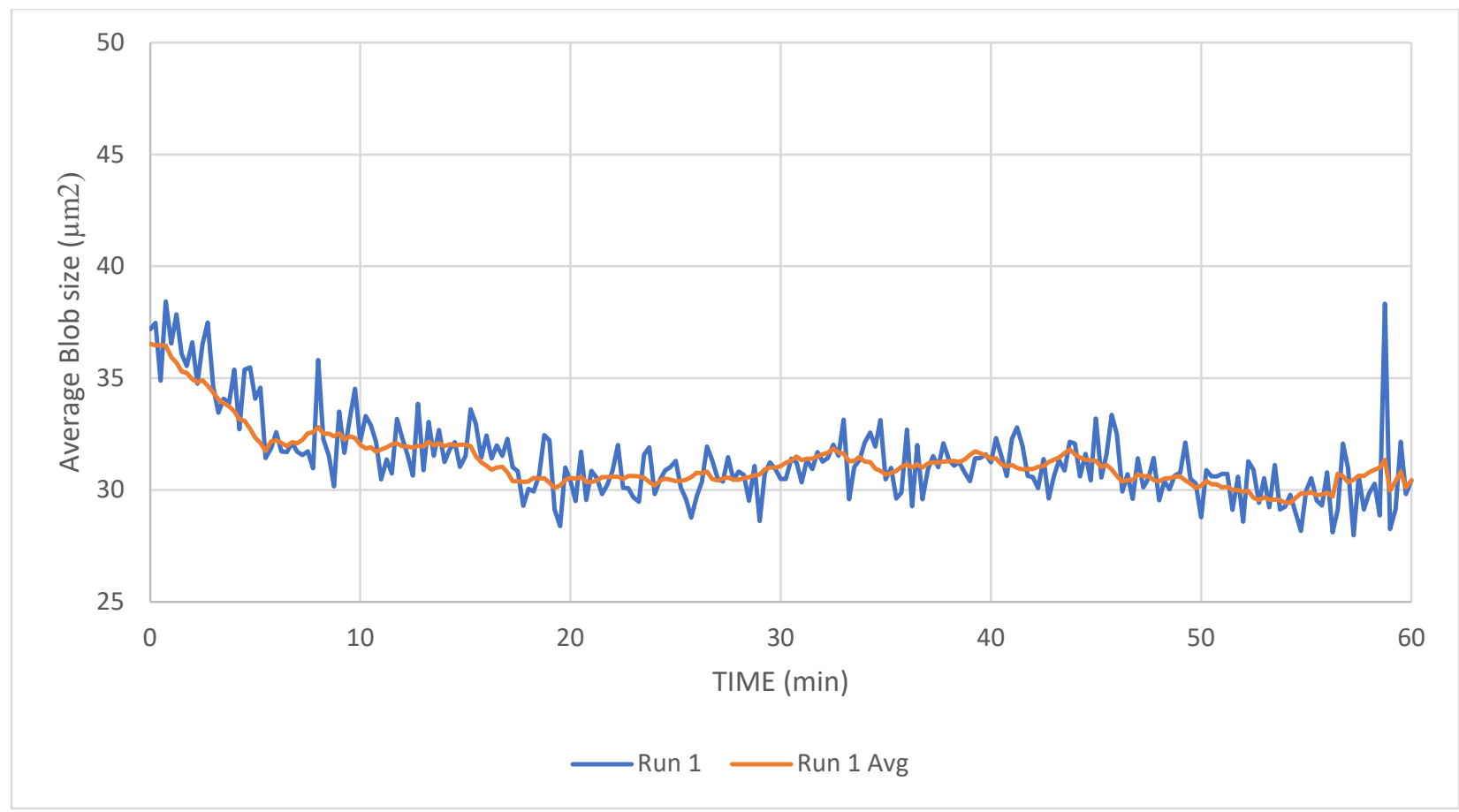

FIGURE 16: BLOB SIZE RESULTS FOR 0.1\% NANOPARTICLE RUN 1

The $0.055 \mathrm{vol} \%$ samples were run in the same fashion as the $0.1 \mathrm{vol} \%$ samples, and the results are shown in Figure 17 below. This run showed variation by no more than $+/-2 \mu \mathrm{m}^{2}$ over the test period. This behavior was mimicked by Runs 2, 5, and 6 . Runs 3 and 4 did not show an increase in blob size but did showed a similar area where the average blob size varied by less than $5 \mu \mathrm{m}^{2}$. 


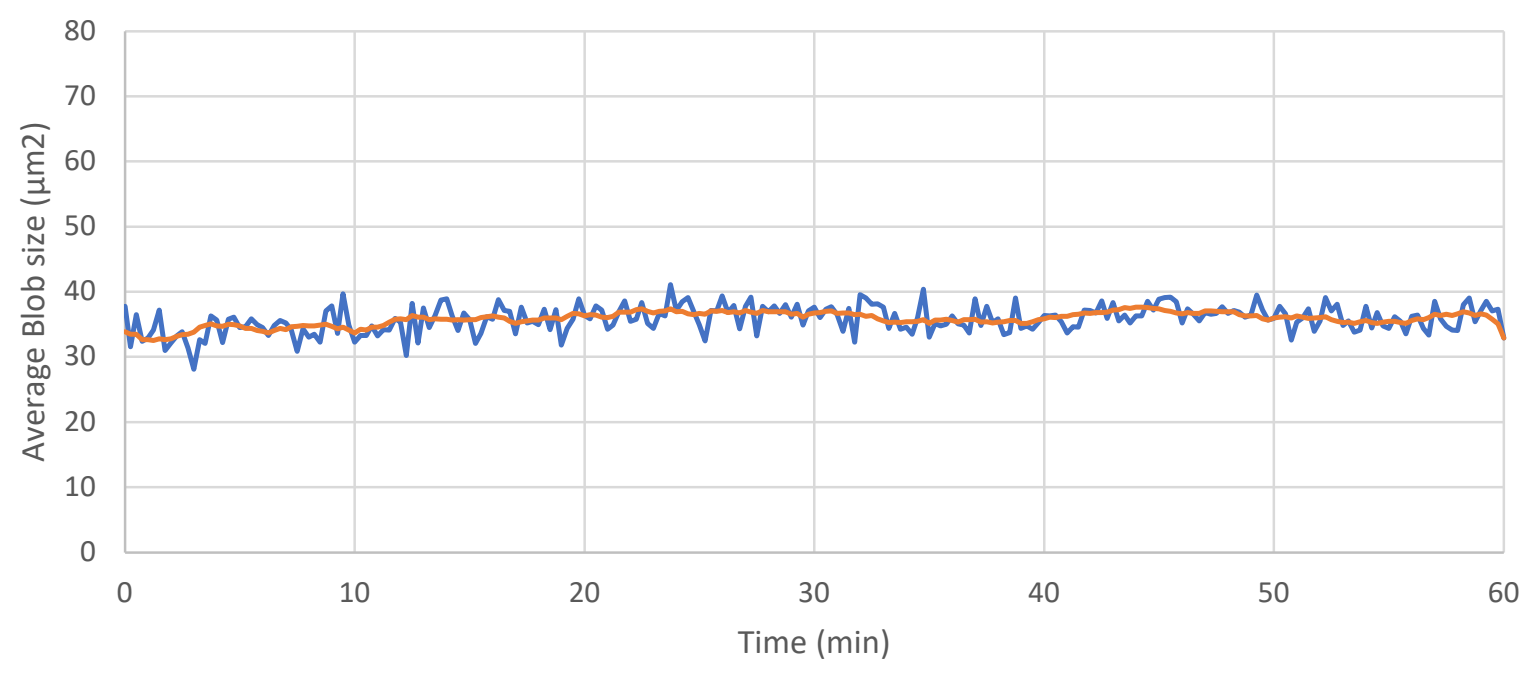

Run $1 \longrightarrow$ Avg

FIGURE 17: BLOB SIZE RESULTS FOR 0.055\% NANOPARTICLE RUN 1

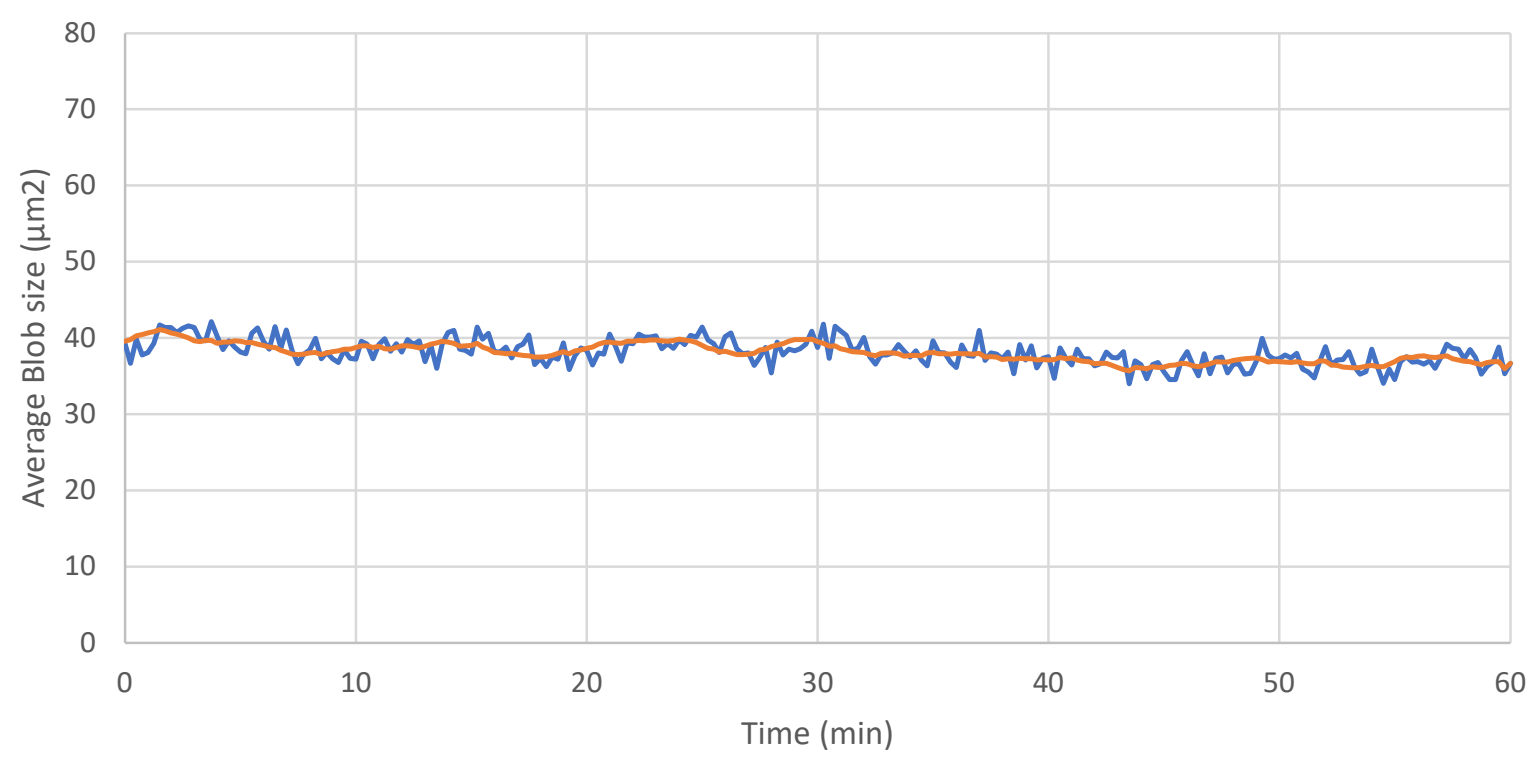

-Run $3 \longrightarrow$ Avg

FIGURE 18: BLOB SIZE RESULTS FOR 0.055\% NANOPARTICLE RUN 3 
Finally, the results were reported for samples with a nanoparticle concentration of 0.01 vol\%. Each of the four samples for this concentration behaved in a similar manner between 35 and $45 \mu \mathrm{m}^{2}$.

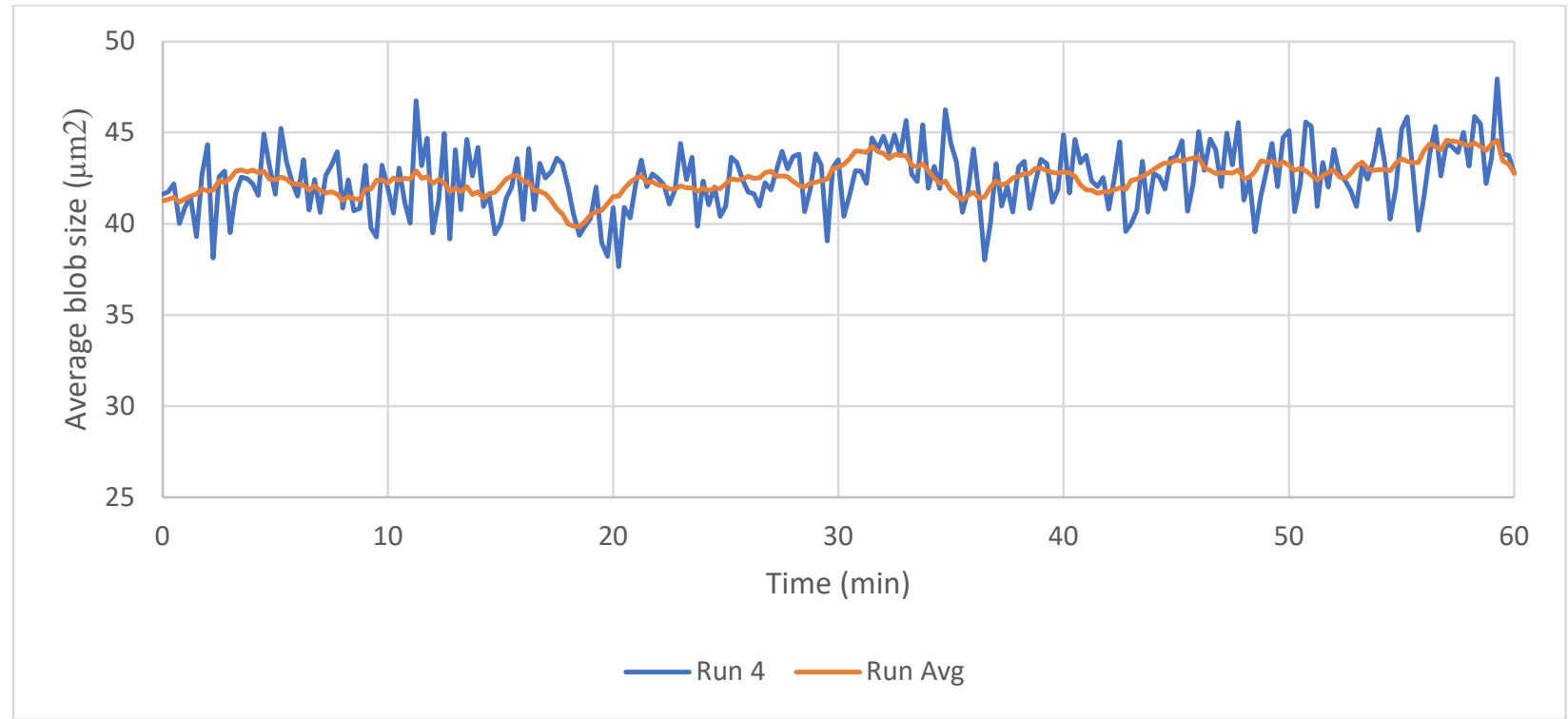

FIGURE 19: BLOB SIZE RESULTS FOR 0.01\% NANOPARTICLE RUN 4

\section{$\underline{\text { Single-Plane Imaging for Microgravity Experiments }}$}

Next, blob size analysis was performed on the 20X single-plane images for nanoparticle mixtures of $0.055 \mathrm{vol} \%$. A representative $20 \mathrm{X}$ image from the SR1 settling run is shown in Figure 20. Samples were prepared for bimodal mixtures with $0.1 \mathrm{vol} \%$ and $0.01 \mathrm{vol} \%$ concentrations, however issues during storage caused these mixtures to develop bubbles within the capillaries, as shown in Figure 21 below. Bubble formation occurred between sample preparation and imaging, likely due to an insufficient seal at the end of each capillary tube. Due to the bubbles, the magnetic stir rod in each capillary was unable to be moved using electromagnetic pulses, and mixing did not occur. For Capillary 2, no bubble formation occurred, and observation proceeded as planned. 


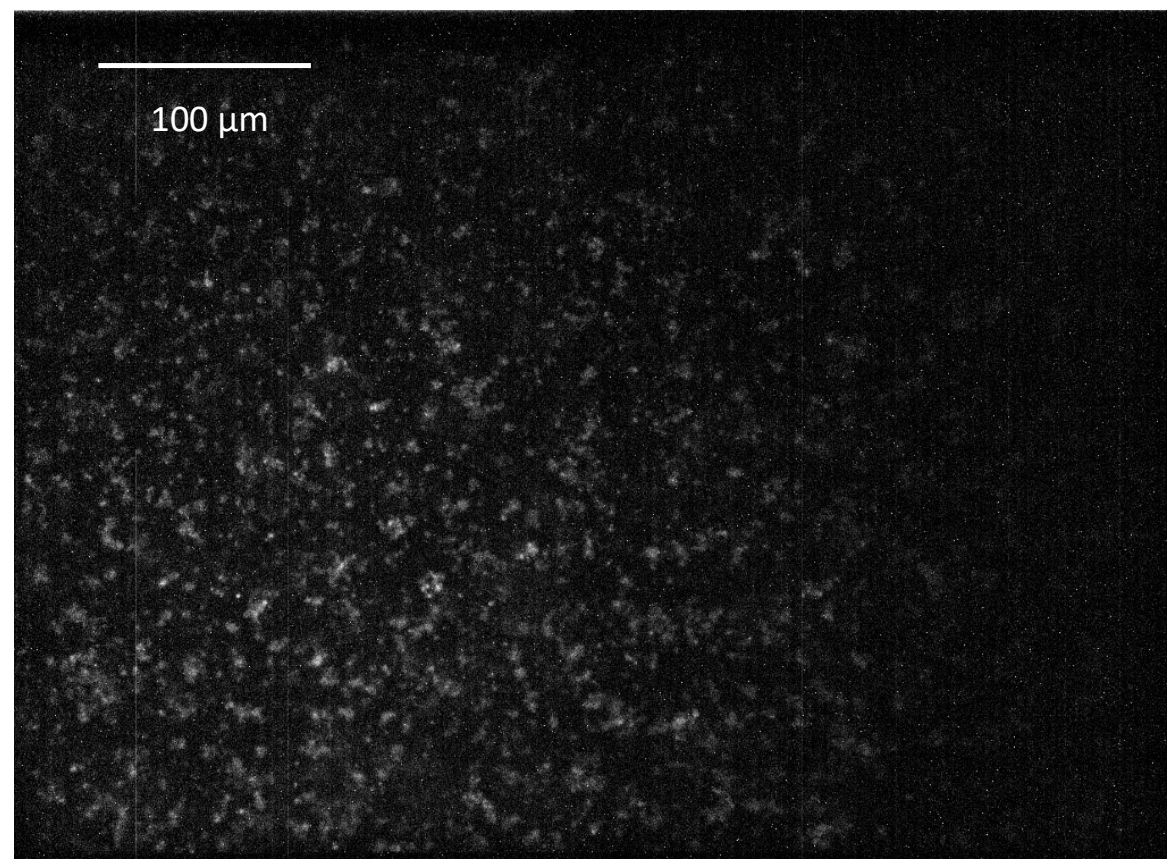

FIGURE 20: A REPRESENTATIVE 20X IMAGE FROM SR1 MICROGRAVITY IMAGING
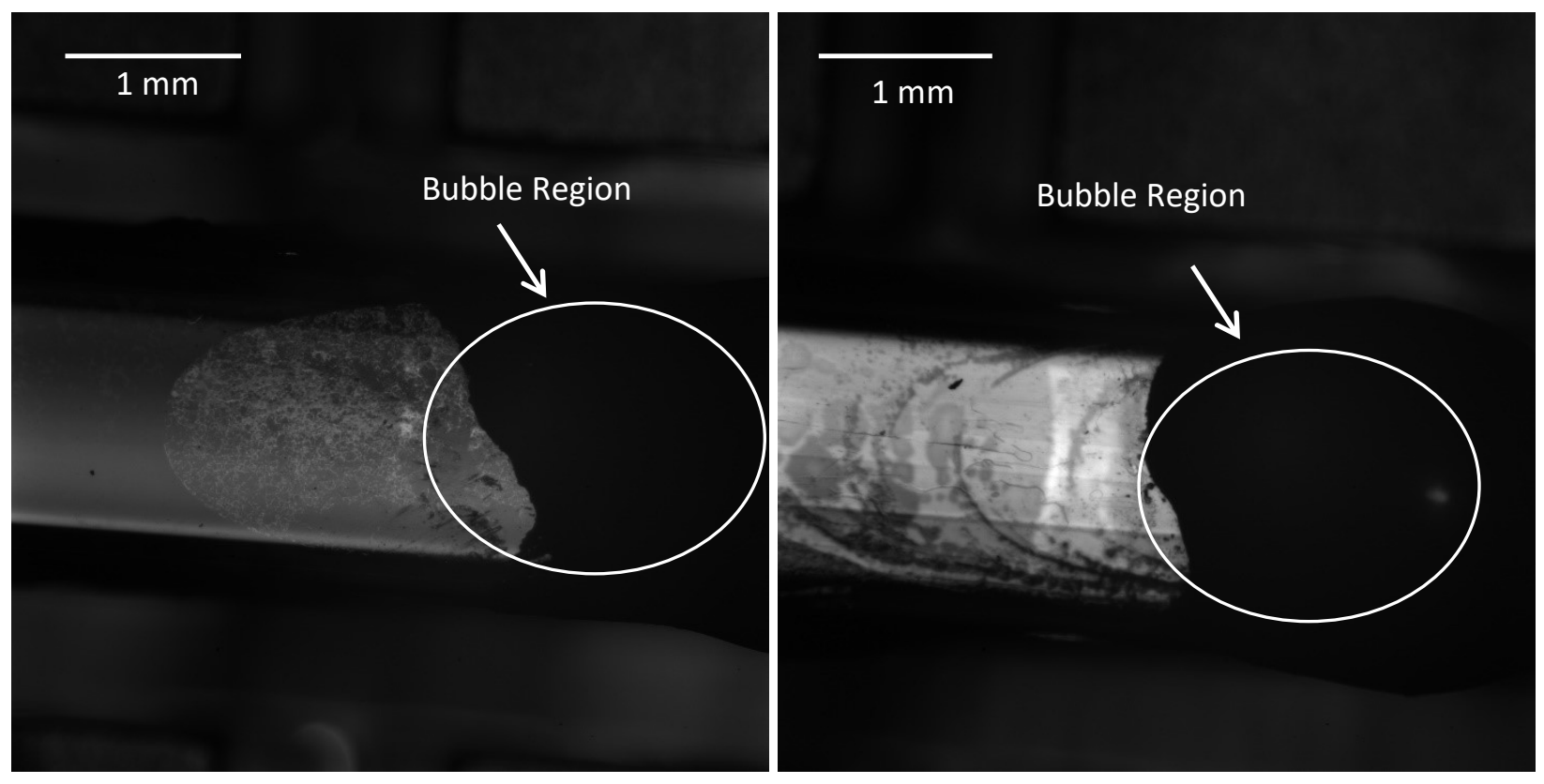

FIGURE 21: 2.5X IMAGE OF CAPPILARY 1 (LEFT) AND CAPPILARY 3 (RIGHT)

SHOWING BUBBLE FORMATION 
Image capture of the 20X images occurred in two phases. Once mixing completed, a fastpaced imaging stage recorded at 10 frames per second for 10 minutes. During the last 50 minutes, images were recorded at 4 frames per minute. This caused a "switching time" of two to three minutes when the first stage was concluded and the second began. For each sample, the initial 10 minutes and final 60 are stitched together.

Figure 22 shows the results from the first 0.055 vol\% Run1. During the last five minutes of the run, the blob size drops from 37 to $27 \mu \mathrm{m}^{2}$. This most likely is an image error, as it is anomalous among the three runs. Finally, Run 1 exhibits a $3 \mu \mathrm{m}^{2}$ increase in blob size during the first two minutes. This most likely is due to an initial agglomeration that occurs after initial mixing, followed by a blob size that maintains around $37 \mu \mathrm{m}^{2}$. 


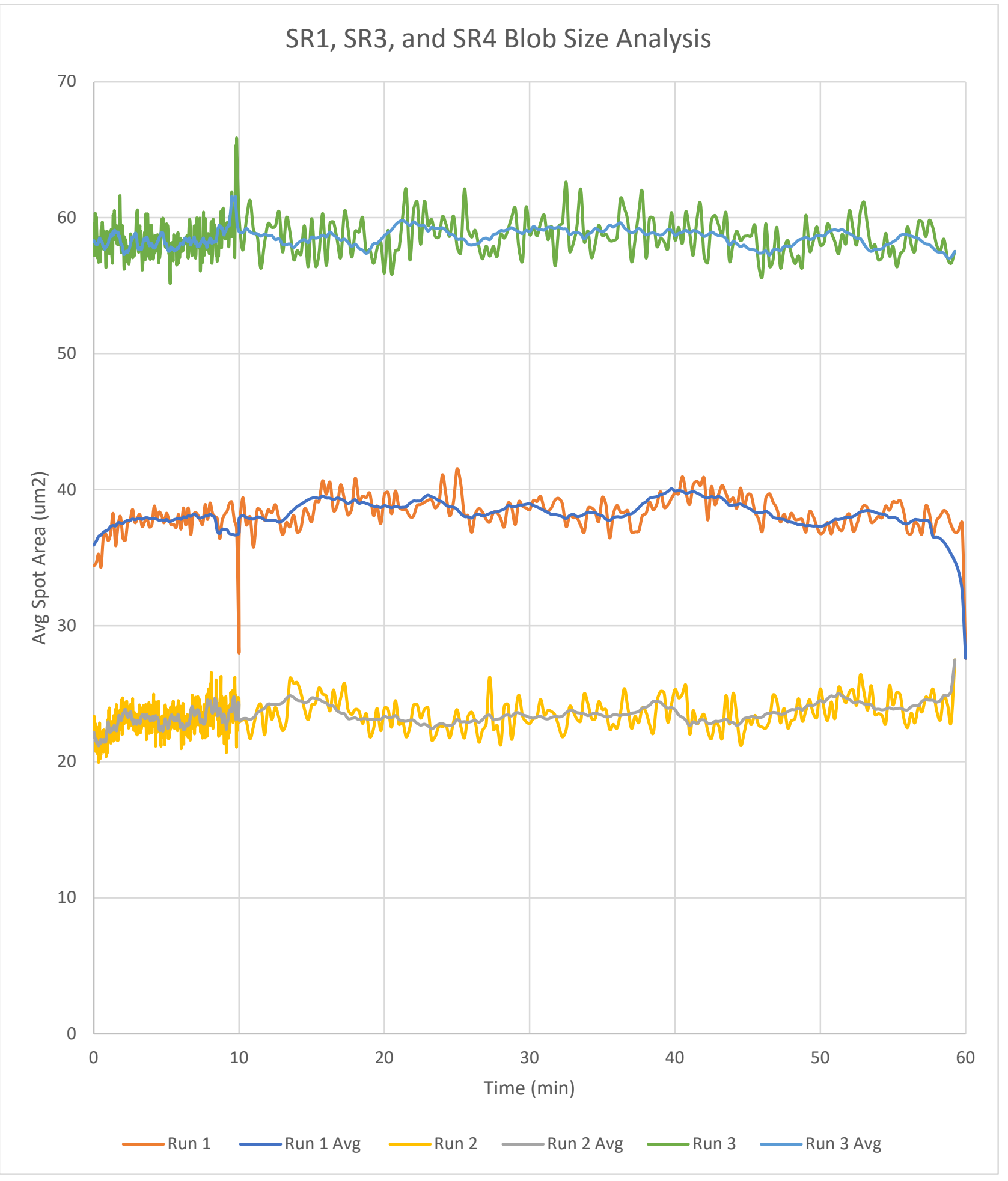

FIGURE 22: BLOB SIZE RESULTS FOR 0.055\% NANOPARTICLE RUNS 1,2, AND 3 
Run 2 (designated as SR3 by NASA) shows much greater consistency, varying by only $+/-3 \mu \mathrm{m} 2$ during its one hour run. Run 2 also exhibited an increase in blob size over the first two minutes like Run 1.

\section{Discussion of the Results}

First, the settling results will be discussed from the $0.1,0.055$ and $0.01 \mathrm{vol} \%$ ground samples. The $0.1 \mathrm{vol} \%$ nanoparticle samples showed a fairly consistent blob size of $35 \mu \mathrm{m}^{2}$ over the hour duration. Why would they behave in this manner? The $0.1 \mathrm{vol} \%$ samples represent the higher-end concentration of nanoparticles, at which the zirconia nanoparticles can potentially adsorb to the surface of the microparticle. Figure 23 below shows the relationship between nanoparticle concentration and nanoparticle adsorption, taken from a similar silica-zirconia system. Based on these results, the system did not experience significant agglomeration over the course of the experiment.

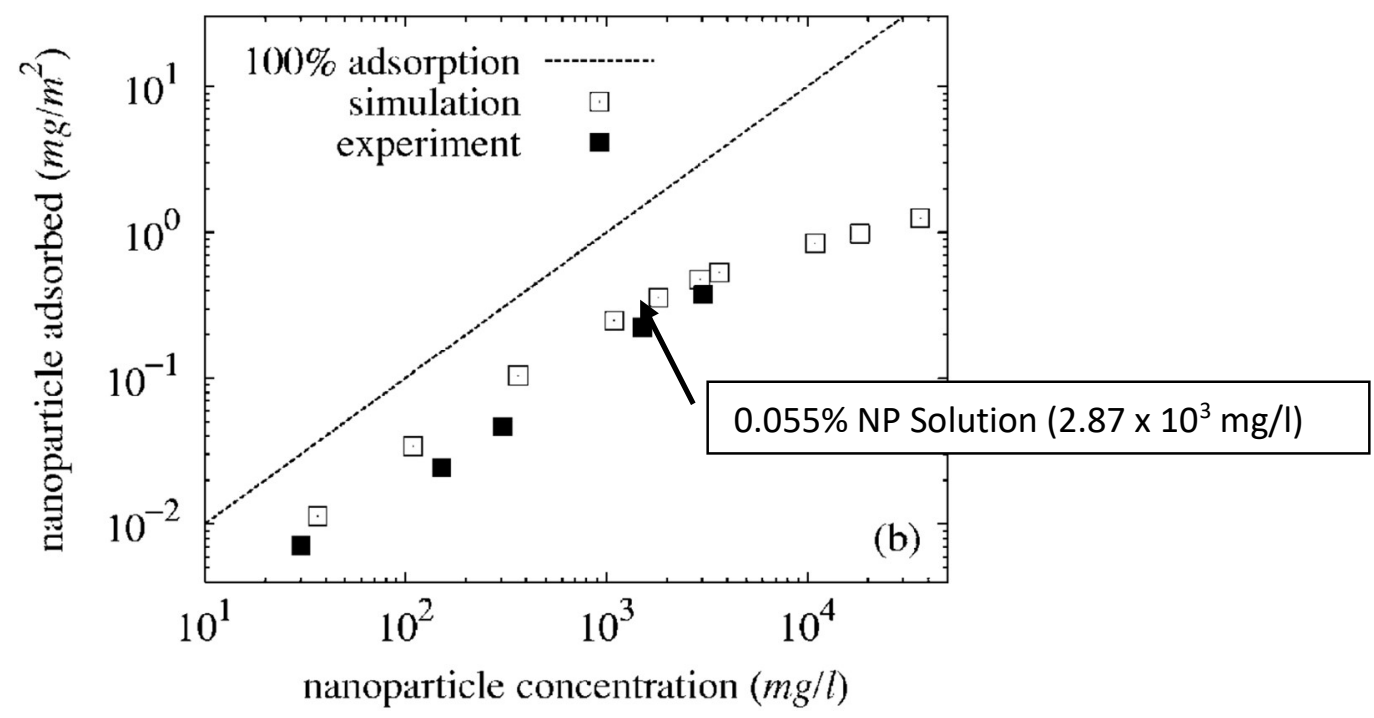

FIGURE 23: NANOPARTICLE ADSORBTION VS. NANOPARTICLE CONCENTRATION

(Liu, 2005) 
The 0.055 vol\% nanoparticle samples showed a marked uniformity in shape, with a gradual increase in blob size until the 30-minute mark. Each ranged between $35 \mu \mathrm{m}^{2}$ and $45 \mu \mathrm{m}^{2}$. These samples represented the ideal zone for nanoparticle haloing samples, as they should be at the correct concentration for haloing to occur. Based on these results, the samples agglomerated slightly before achieving a thermodynamic homeostasis. Given the consistency of these results, the samples appear to have been stabilized by the mechanism of nanoparticle haloing.

Finally, the 0.01 vol\% nanoparticle samples experienced a small decrease in blob size followed by a flatline. These samples represent the lower end of nanoparticle concentration. At this concentration, there are insufficient amounts of nanoparticles to provide a significant repulsive effect. While the $0.01 \mathrm{vol} \%$ sample was expected to undergo additional agglomeration and a higher blob size than the 0.055 vol\% sample, it did not show significant agglomeration over the duration of the experiment.

\section{Comparison of Ground and ISS results.}

The microgravity samples differed in magnitude, yet their blob size results all showed consistency in their shape. Each sample held a blob size within $+/-3 \mu \mathrm{m}^{2}$ over the course of the experiment. In contrast, the ground results for $0.055 \mathrm{vol} \%$ nanoparticle haloing systems behaved slightly differently. While the samples did flatline towards the end of the experiment, most increased in blob size until the 30-minute mark. This result seems counter-intuitive, as the ground samples should agglomerate more quickly under the influence of gravity. Why would the microgravity samples take less time to aggregate? This is most likely due to the difference in mixing. Each ground sample was mixed using high energy sonication. This method is the preferred means for mixing, as it breaks up microparticles evenly. The mixing on board the ISS uses a slowly-moving magnetic mixing bar, which most likely resulted in lesser-dispersed 
particle systems. Thus, the microgravity samples would take less time to find equilibrium than the sonicated ground particles

Why did the microgravity results differ so greatly from each other in magnitude? One influence was the lighting conditions for the experiment. One consideration for this study was the effect of bleaching on the nanoparticles. From preliminary ground testing, the microparticles were shown to bleach under the illumination of the $532 \mathrm{~nm} \mathrm{Nd}$ laser. For this reason, the laser was partially shuttered during SR3 testing. This reduced the illumination by a factor of 20 and dimmed the resultant image. The ImageJ macro has a built-in threshold to even out the brightness of images, however when the illumination decreases to such a degree the program reads the edges of the blobs to be smaller than they are in actuality. An example of the image dimming is shown in Figure 24 below. While the program attempts to compensate for this dimming, the result is a smaller blob size than expected. Likewise, the increase in blob size from SR1 to SR3 can be attributed to an increase in illumination brightness. This occurred when no significant bleaching was observed during SR3, and the brightness was increased beyond its initial SR1 starting point.

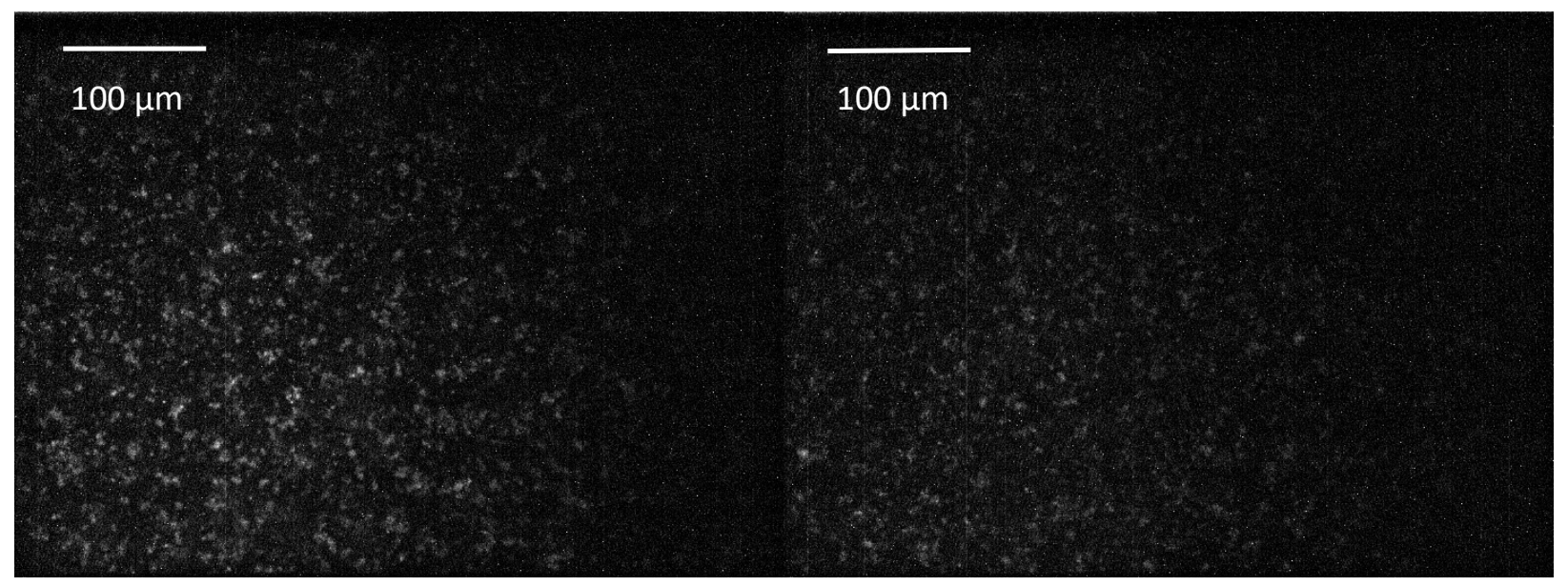




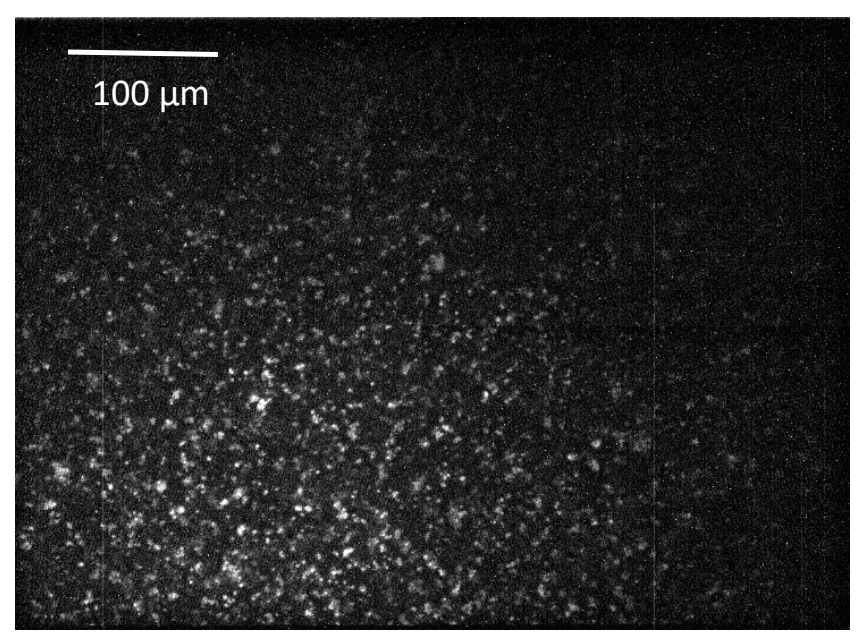

FIGURE 24: DIFFERENCE IN ILLUMINATION BETWEEN SR1 (LEFT) SR3 (RIGHT) AND

$$
\text { SR4 (BOTTOM) }
$$

\section{Sources of Error}

There were several sources of error within this experiment. Beyond the illumination uniformity, issues arose while setting the threshold level for the blob size program. When the blob size is calculated, the program must set a brightness level at which it distinguishes a blob from the background. If the brightness threshold for the blob is increased, the calculated blob size is decreased. To mitigate this effect, the program calculated the threshold based on the given brightness value of the image.

Another source of error arose due to the mixing method. Currently, the only mixing available on the light microscopy module is by way of micro-stirring rods using electromagnetic pulses. These pulses both turn the rod and translate it back and forth across the capillary tube. Conversely, the ground experiments used sonication to mix the colloidal samples. Sonication is a high-energy process (500 watts for our Qsonica machine), and thus is more likely to break down existing clumps of particles than the ACE-T stirring method. To mitigate the difference in 
mixing, each sample was checked using a $2.5 \mathrm{X}$ magnification to ensure it was fully mixed. Additionally, the microgravity experiments included a delay between the conclusion of the mixing script and the beginning of the imaging script. This delay lasted between 5-15 minutes, meaning that some agglomeration could occur before the first images were captured.

\section{Difficulties}

The following include recommendations for future nanoparticle haloing experiments to be conducted in a similar manner on the ISS in future experiments. This section reviews issues encountered while performing the experiment, as well as later during image acquisition. For each difficulty encountered, a recommendation is provided to improve the experiment.

The first issue arose during the initial imaging of the capillary tubes. The initial $2.5 \mathrm{X}$ imaging revealed that Capillaries 1 and 3 had large bubbles within them. This could be attributed to the extended stow period between the capillary sealing and use (five months stowed on board the launch vehicle and in the ISS). The issue could also be a result of a sealing error during capillary preparation, since Capillary 2 did not have a similar issue.

The second issue came during the modified-temperature experiments, a series of tests done after the research in this experiment. During these experiments, the temperature was varied from roughly ambient temperature $\left(\sim 23^{\circ} \mathrm{C}\right)$ to as high as $55^{\circ} \mathrm{C}$. Due to these temperature fluctuations, Capillary 2 developed an abnormality, a small crack roughly in the center of the tube. This fissure can be seen in Figure 25 on the right side of the image. Over the course of two 
days, atmosphere from the ISS seeped into the capillary and further experiments were discontinued.

The final issue occurred while downloading the files. Due to the method of imaging and the number of trials run, the total quantity of files amounted to 400,000 images. Each image was 8-bit, 2.39 megabytes and comprised of 1824 x 1340 pixels, which took up 885 Gigabytes. This took an extended time to download from the NASA servers, which have a download speed of 5 $\mathrm{MB} / \mathrm{sec}$.

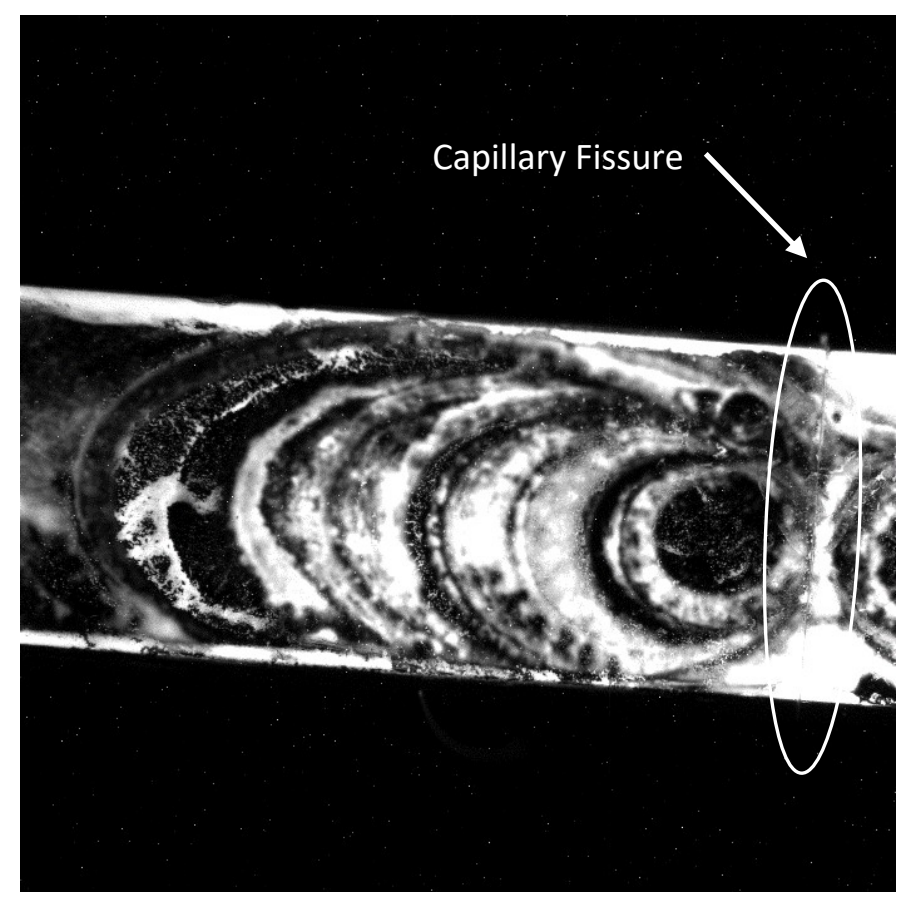

FIGURE 25: DEVELOPMENT OF FISSURE IN CAPILLARY 2

Below are a few changes that can be made for the future experiments. To combat the bubble issue, the capillaries should be filled a shorter time before testing. This will prevent the most diffusion of air into the tubes. Additionally, future researchers should confer with NASA to ensure proper sealing of the tubes during preparation. 
To avoid cracking, future researchers should change the construction of the capillary tubes. The current design of the system includes a copper heat pipe which is glued directly to the glass capillary. During temperature experiments the difference in the thermal expansion coefficient between the copper and the glass caused stress on the capillary, causing it to fracture. Future designs should include a heat-conductive layer that expands and contracts with the glass, eliminating the stress. Possible designs include a heat-conducting grease between the copper tube and the capillary. This will allow the copper and glass to expand independently, preventing future fracturing from occurring.

Finally, the total file size of the download should be reduced by lowering the image capture rate. Each image took 2.39 MB of disk space, and at a rate of 10 images per second each 10-minute capture period took 14.22 Gigabytes of space. In comparison, the latter 50 minutes of the experiment took less than $0.5 \mathrm{~Gb}$. To combat this and simplify the image capture, I recommend switching to a uniform four images/minute rate for the entire hour period. This will shrink the file size per run to $568 \mathrm{MB}$. 


\section{CONCLUSIONS}

In conclusion, this study focused on the behavior of colloidal mixtures with varying concentrations of nanoparticles in order to examine the effects of concentration and microgravity on bimodal microparticle-nanoparticle mixtures. The study found that both the $0.055 \mathrm{vol} \%$ ground samples and the $0.055 \mathrm{vol} \%$ ISS samples achieved an equilibrium at some point in the run, however the ground samples reached equilibrium at around 30 minutes compared to the ISS samples which stabilized within three minutes into the run.

The study encountered several issues while observing the ISS samples in this experiment. Due to poor capillary seals, Capillaries 1 and 3 were filled with bubbles of vapor that stopped any form of mixing from occurring. Differing lighting conditions altered the blob size results between SR1, SR3, and SR4. Finally, a high image capture rate caused the download size to balloon to 885 Gigabytes of space, which slowed the data analysis. These concerns were noted and mitigated as much as possible. Suggestions were given for future microgravity colloidal experiments to improve these key areas. This study noted key factors for colloidal systems in microgravity and laid the groundwork for future nanoparticle haloing experiments. 


\section{REFERENCES}

Hassan Azzazy, Mai Mansour. 2009. In vitro diagnostic prospects of nanoparticles. Science Direct 403: 1-8

Robert Brown. 1828. A brief Account of Microscopical Observations made in the Months of June, July, and August, 1827, on the Particles contained in the Pollen of Plants; and on the general Existence of active Molecules in Organic and Inorganic Bodies. Philosophy Magazine 161-173

Carey, G. H., Abdelhady, A. L., Ning, Z., Thon, S. M. Bakr, O. M., and Sargent, E. H. 2015. Colloidal Quantum Dot Solar Cells. American Chemical Society. Chemical Review 23: 32-63

Albert Einstein. 1905. Über die von der molekularkinetischen Theorie der Wärme geforderte Bewegung von in ruhenden Flüssigkeiten suspendierten Teilchen. Annalen der Physik 322: 549560

Yijun Gao, Jiang Zheng, Weijian Chen, Lin Yuan, Zhi Teh, Jianfeng Li Yang, Xin Cui, Gavin Conibeer, Robert Patterson, Shujuan Huang. 2019. Enhancing PbS Colloidal Quantum Dot Tandem Solar Cell Performance by Graded Band Alignment. The Journal of Physical Chemistry Letters 19: 5729-5734

Gerhard Gompper, Michael Schick. 2007. Soft Matter: Colloidal order: entropic and surface forces Wiley Online Library. Wiley

Thomas Graham. 1861. Liquid Diffusion Applied to Analysis. Philosophical Transactions of the Royal Society of London 151: 183-224

Hugo Hamaker. 1937. The London—van der Waals attraction between spherical particles. Physica 10: 1058-1072 
Qingwen He. 2014. Investigation of Stabilization Mechanisms for Colloidal Suspension Using Nanoparticles. J. B. Speed School of Engineering, University of Louisville

Xiaoting Hong. 2009. Direct Force Measurement for the Silica-Plate System in Nanoparticle Suspensions by Colloidal Probe Technique. J. B. Speed School of Engineering, University of Louisville.

L. A. Hough, A. M. Alsayed, C. Badre, R. Dreyfus. 2013. Physics of Complex Colloids. Proceedings of the International School of Physics.

Elizabeth Howell. “International Space Station: Facts, History \& Tracking” Spaceflight. February 08, 2018 https://www.space.com/16748-international-space-station.html Accessed November 23rd, 2019.

Robert J. Hunter. 1981. Zeta Potential in Colloid Science: Principles and Applications. School of Chemistry, University of Sydney. Academic Press.

J. Israelachvili. 2015. Intermolecular and Surface Forces (3rd Edition). Academic Press

Raz Jelinek. 2009. Cellular and Biomolecular Recognition: Synthetic and non-Biological Molecules. Wiley-VCH, Weinheim

Jorg Kreuter. 1994. Colloidal Drug Delivery Systems. Marcel Dekker Inc. Madison Avenue, NY.

Ajeet Kumar. 2017. Methods for characterization of Nanoparticles. Science Direct. 8: 232-248

Evgenii Mikhailovich Lifshitz. 1992. Perspectives in Theoretical Physics. Science Direct 392443

Erik Luijten and Jiwen Liu. 2005. Colloidal stabilization via nanoparticle halo formation. Physical Review 72: 291-301 
D. H. Napper. 1976. Steric Stabilization. Science Direct. 58: 81-99

Niharika Neerudu, Louis McNamara, Nathan I. Hammer, and Hemali Rathnayake. 2017. A versatile synthesis to novel binary reactive groups. Science Advances Today 25: 266-272

A. J. Nozik. 2002. Quantum Dot Solar Cells. Physica E. 14: 115-120

Timothy Reckart. "The Light Microscopy Module (LMM)." Glenn Research Center. June 12, 2019 https://www1.grc.nasa.gov/space/iss-research/iss-fcf/fir/lmm/lmm-brochure/. November $25^{\text {th }}, 2019$.

Sydney Ross and Ian D. Morrison. 1988. Colloidal Systems and Interfaces. Wiley-Interscience Sven Ruhle. 2016. Tabulated values of the Shockley-Queisser limit for single junction solar cells. Solar Energy 130: 139-147

Everett N. Scheer, K. S. S. 2008. A phase diagram of attractive microspheres and repulsive nanoparticles. The Journal of Chemical Physics 128: 62-88

Francesco Selmi. 1845. Intorno all'azione dell'iodio sopra il clorido di mercurio: memoria prima di Francesco Selmi. Milano

Niharika Sreeramulu. 2016. Colloidal Self-Assembly of Multi-fluorescent Silsesquioxane Microparticles. The Department of Chemistry, Western Kentucky University

Valeria Tohver, James E. Smay, Alan Braem, Paul V. braun, Jennifer A. Lewis. 2001. Nanoparticle halos: A new colloid stabilization mechanism. PNAS 98: 8950-8954

Adam Vrij and Nieuwen Huis. 1978. Application of modern concepts in liquid state theory to concentrated particle dispersions. Faraday Discussions of the Chemical Society 65: 101-113 


\section{APPENDIX I. ZETA POTENTIAL RESULTS FOR SSQ BATCHES}

\begin{tabular}{|c|c|c|}
\hline \multicolumn{3}{|c|}{ BC-SSQ (5) } \\
\hline Date & $\mathrm{pH}$ & ZP \\
\hline $6 / 13 / 2018$ & 3.1 & -16.512 \\
\hline $6 / 13 / 2018$ & 3.2 & -13.128 \\
\hline $6 / 26 / 2018$ & 3.2 & -6.109 \\
\hline $6 / 13 / 2018$ & 3.3 & -29.613 \\
\hline $6 / 25 / 2018$ & 3.3 & -13.471 \\
\hline $7 / 9 / 2018$ & 4 & -28.211 \\
\hline $7 / 17 / 2018$ & 4 & -22.809 \\
\hline
\end{tabular}

\begin{tabular}{|c|c|c|}
\hline \multicolumn{3}{|c|}{ BC-SSQ (6) } \\
\hline Date & $\mathrm{pH}$ & ZP \\
\hline $7 / 17 / 2018$ & 4 & -27.731 \\
\hline $7 / 20 / 2018$ & 3.952 & -41.053 \\
\hline
\end{tabular}

\begin{tabular}{|c|c|c|}
\hline \multicolumn{3}{|c|}{ BC-SSQ (7) } \\
\hline Date & $\mathrm{pH}$ & ZP \\
\hline $7 / 17 / 2018$ & 4 & -27.637 \\
\hline
\end{tabular}

\begin{tabular}{|c|c|c|}
\hline \multicolumn{3}{|c|}{ BC-SSQ (8) } \\
\hline Date & $\mathrm{pH}$ & ZP \\
\hline $6 / 13 / 2018$ & 3.1 & -11.333 \\
\hline $6 / 13 / 2018$ & 3.2 & -13.675 \\
\hline $6 / 26 / 2018$ & 3.2 & 8.482 \\
\hline $6 / 13 / 2018$ & 3.3 & 2.2645 \\
\hline $6 / 25 / 2018$ & 3.3 & -14.939 \\
\hline $7 / 17 / 2018$ & 4 & -32.777 \\
\hline
\end{tabular}

\begin{tabular}{|c|c|c|}
\hline \multicolumn{3}{|c|}{ BC-SSQ (9) } \\
\hline Date & pH & ZP \\
\hline $7 / 17 / 2018$ & 4 & -31.054 \\
\hline $7 / 20 / 2018$ & 3.952 & -28.662 \\
\hline
\end{tabular}




\begin{tabular}{|c|c|c|}
\hline \multicolumn{3}{|c|}{ BC-SSQ (10) } \\
\hline Date & $\mathrm{pH}$ & ZP \\
\hline $7 / 24 / 2018$ & 3.055 & -14.095 \\
\hline $6 / 13 / 2018$ & 3.1 & 12.246 \\
\hline $6 / 13 / 2018$ & 3.2 & 7.678 \\
\hline $6 / 26 / 2018$ & 3.2 & 9.321 \\
\hline $6 / 13 / 2018$ & 3.3 & -12.535 \\
\hline $6 / 25 / 2018$ & 3.3 & -14.036 \\
\hline $7 / 24 / 2018$ & 3.67 & -39.319 \\
\hline $7 / 17 / 2018$ & 4 & -23.201 \\
\hline $7 / 20 / 2018$ & 3.952 & -31.365 \\
\hline
\end{tabular}

\begin{tabular}{|c|c|c|}
\hline \multicolumn{3}{|c|}{ BC-SSQ (11) } \\
\hline Date & $\mathrm{pH}$ & ZP \\
\hline $7 / 24 / 2018$ & 3.055 & -8.286 \\
\hline $7 / 24 / 2018$ & 3.67 & -36.682 \\
\hline $7 / 9 / 2018$ & 4 & -4.722 \\
\hline $7 / 9 / 2018$ & 4 & -8.44 \\
\hline $7 / 17 / 2018$ & 4 & -26.33 \\
\hline $7 / 20 / 2018$ & 3.952 & -30.202 \\
\hline
\end{tabular}

\begin{tabular}{|c|c|c|}
\hline \multicolumn{3}{|c|}{ BC-SSQ-RB (12) } \\
\hline Date & $\mathrm{pH}$ & ZP \\
\hline $8 / 27 / 2018$ & 3.12 & -11.291 \\
\hline $8 / 27 / 2018$ & 3.44 & -25.59 \\
\hline $8 / 27 / 2018$ & 3.77 & -32.519 \\
\hline $8 / 27 / 2018$ & 4.03 & -32.4878 \\
\hline $8 / 28 / 2018$ & 2.72 & 19.025 \\
\hline $8 / 29 / 2018$ & 2.87 & 4.979 \\
\hline
\end{tabular}

\begin{tabular}{|c|c|c|}
\hline \multicolumn{3}{|c|}{ BC-SSQ (12) } \\
\hline Date & $\mathrm{pH}$ & ZP \\
\hline $8 / 27 / 2018$ & 3.12 & -14.5433 \\
\hline $8 / 27 / 2018$ & 3.44 & -27.1656 \\
\hline $8 / 27 / 2018$ & 3.77 & -28.4444 \\
\hline $8 / 27 / 2018$ & 4.03 & -32.4956 \\
\hline $8 / 28 / 2018$ & 2.72 & 14.746 \\
\hline $8 / 29 / 2018$ & 2.87 & 5.994 \\
\hline
\end{tabular}


APPENDIX II: GROUND BLOB SIZE ANALYSIS RESULTS

0.1 Volume Percent Bimodal Colloidal Mixture
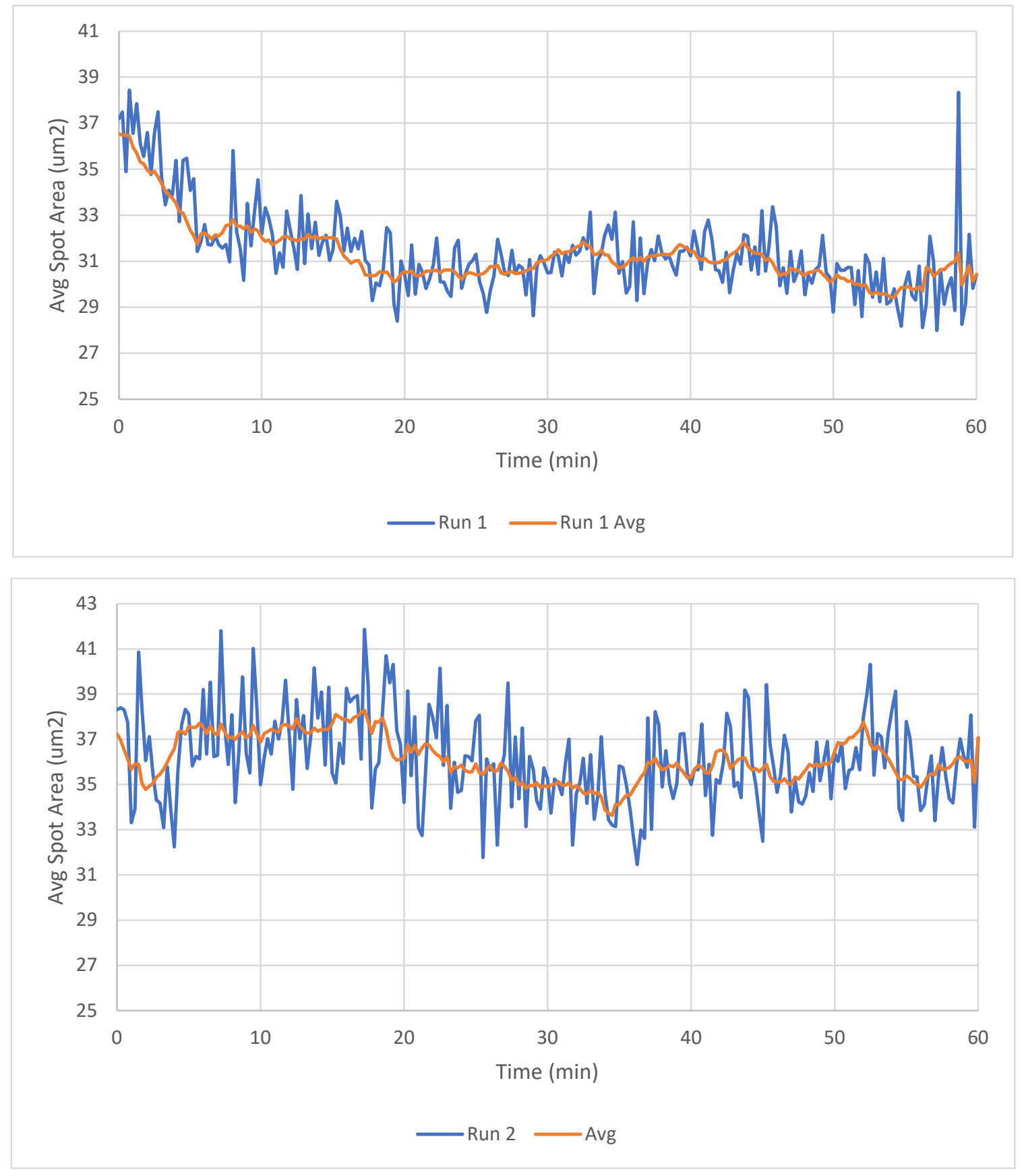


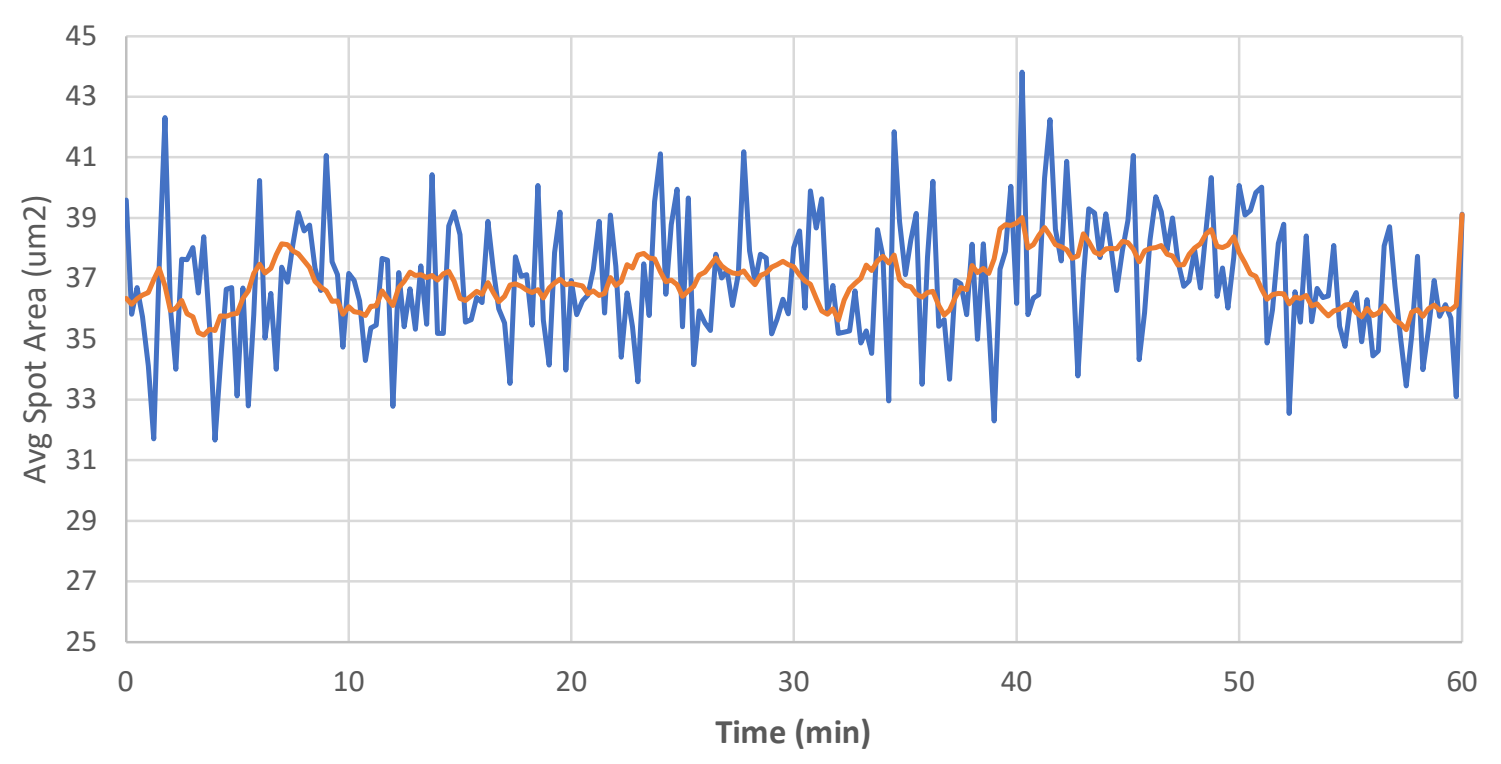

- Run $3 \longrightarrow$ Run Avg

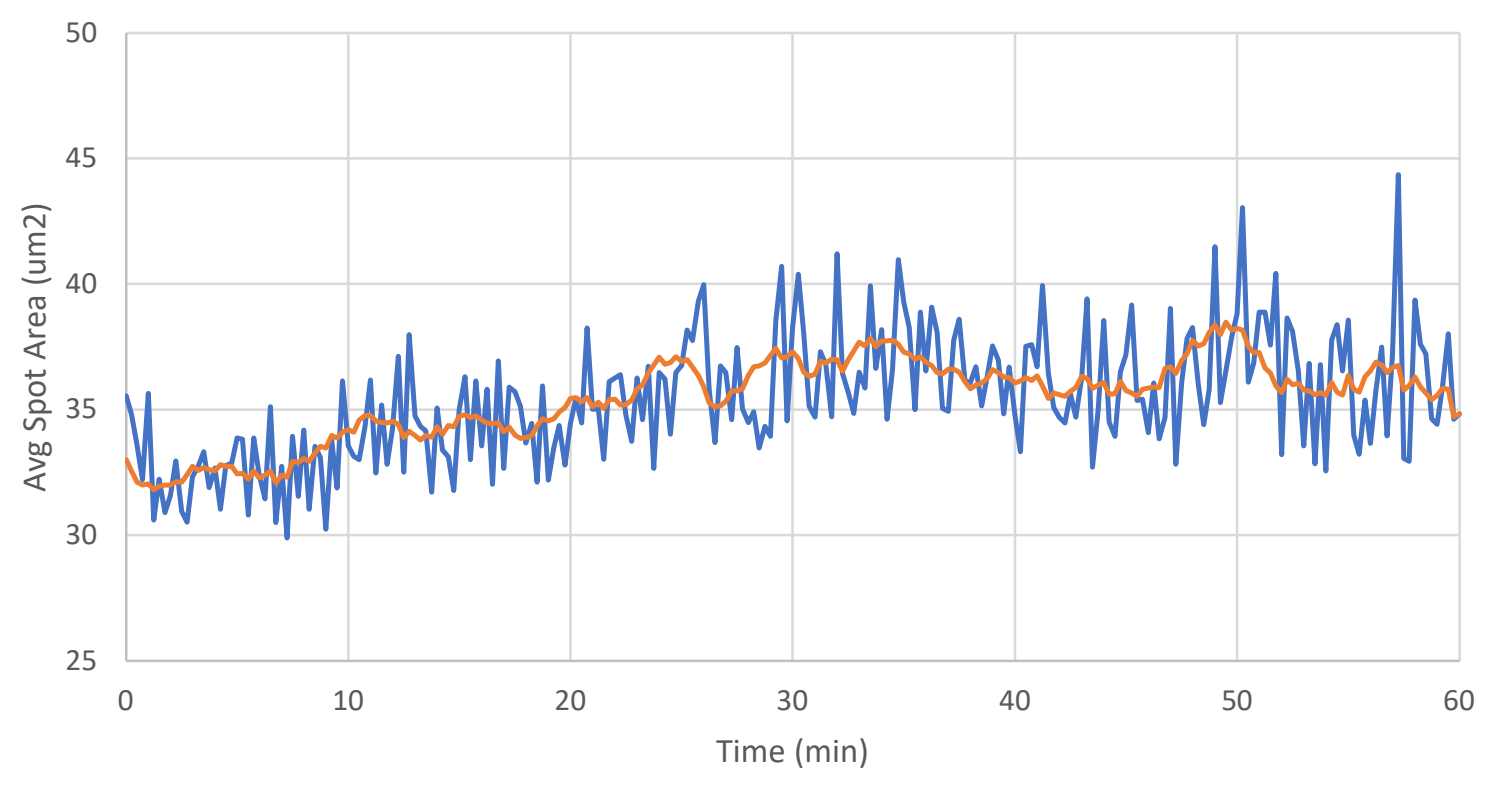

Run 4 Run Avg 

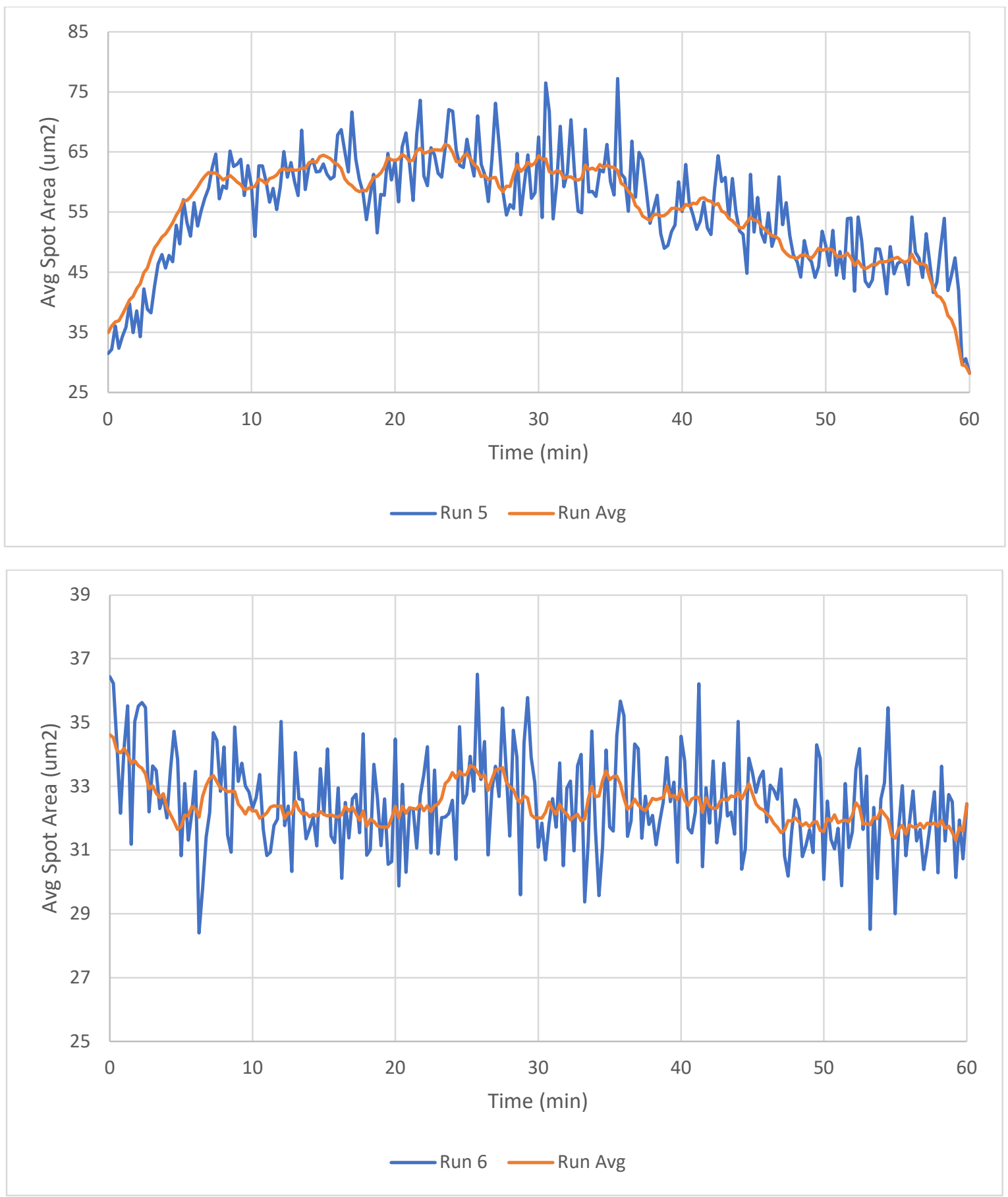


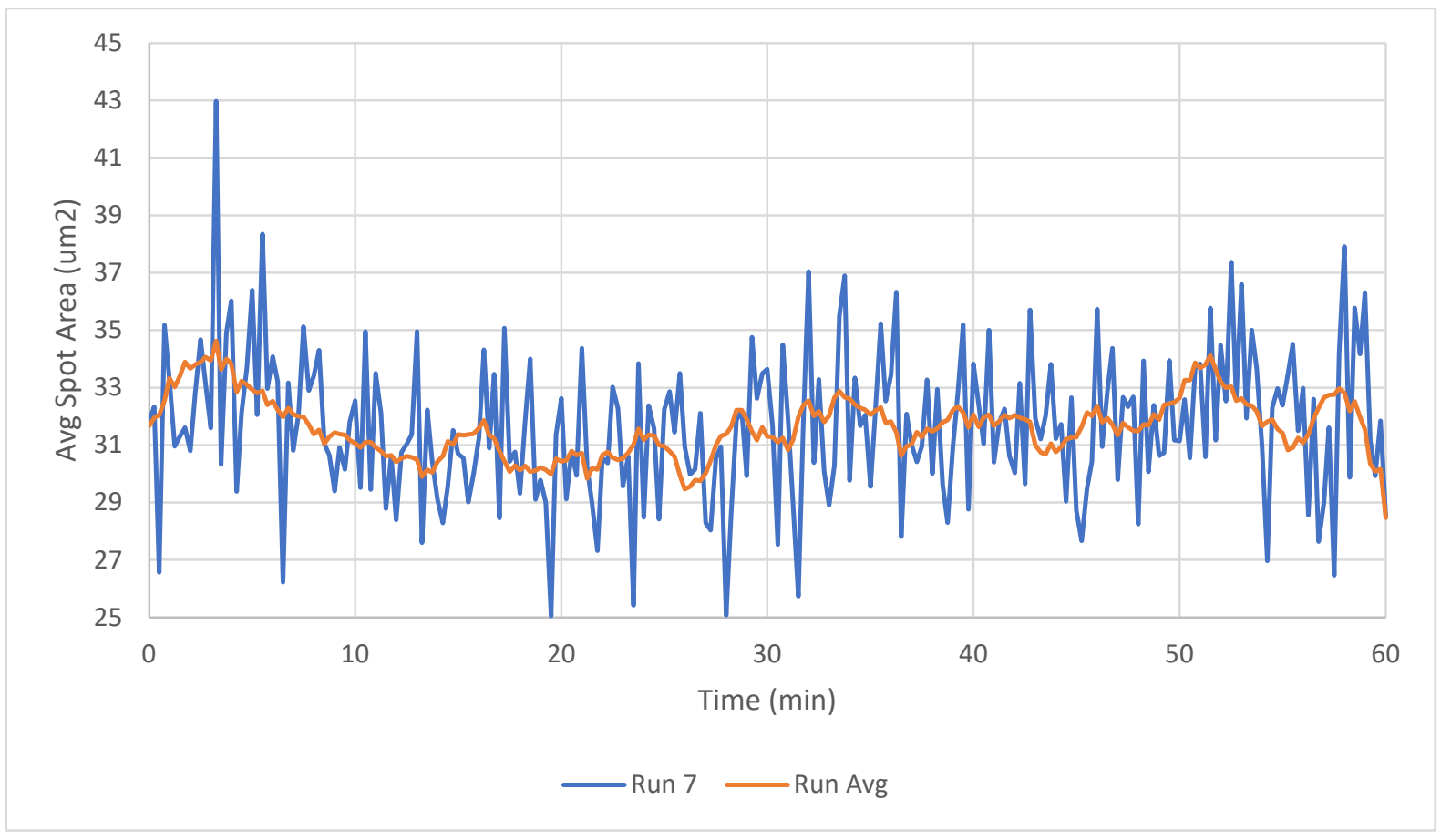

0.055 Volume Percent Bimodal Colloidal Mixture

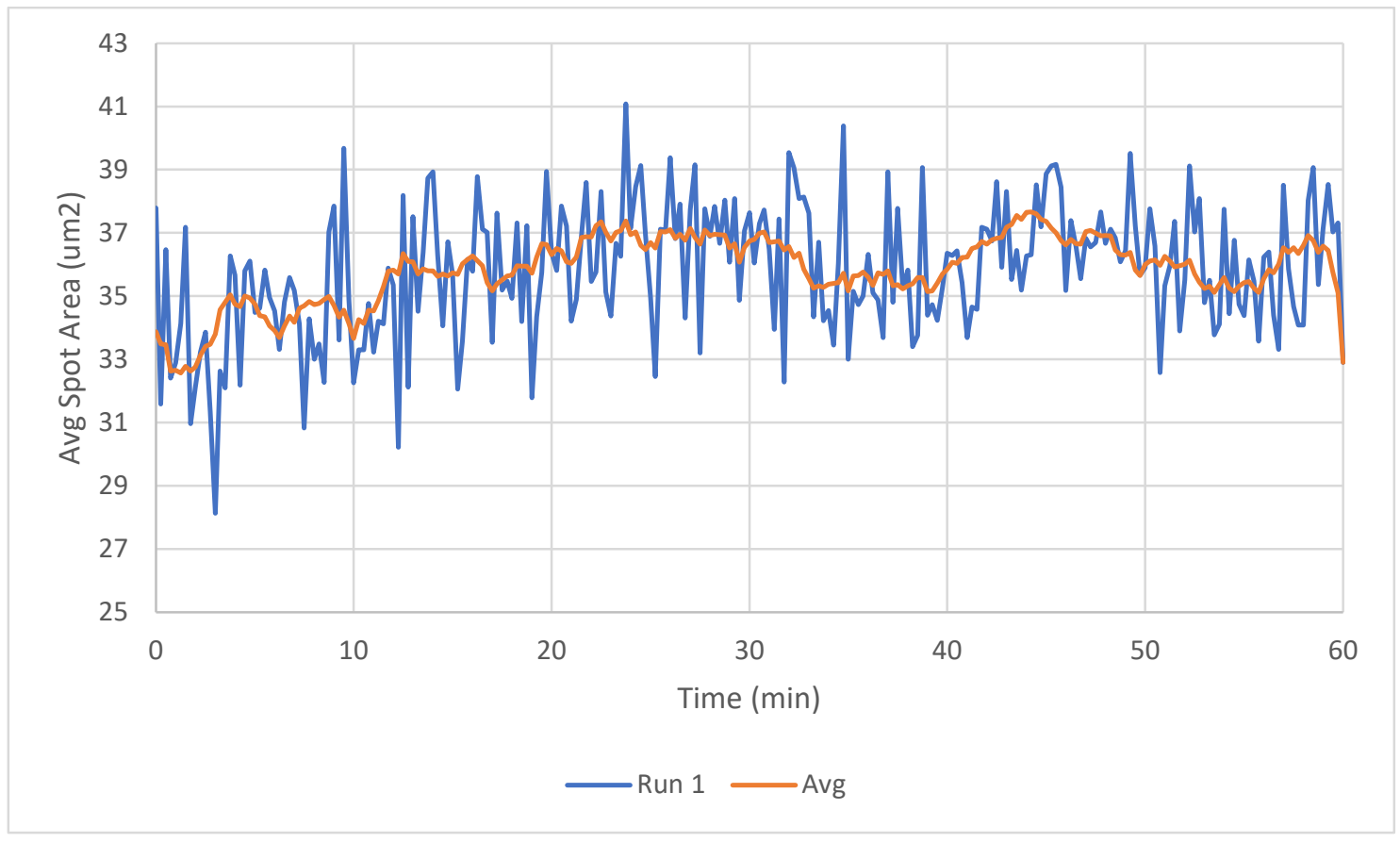



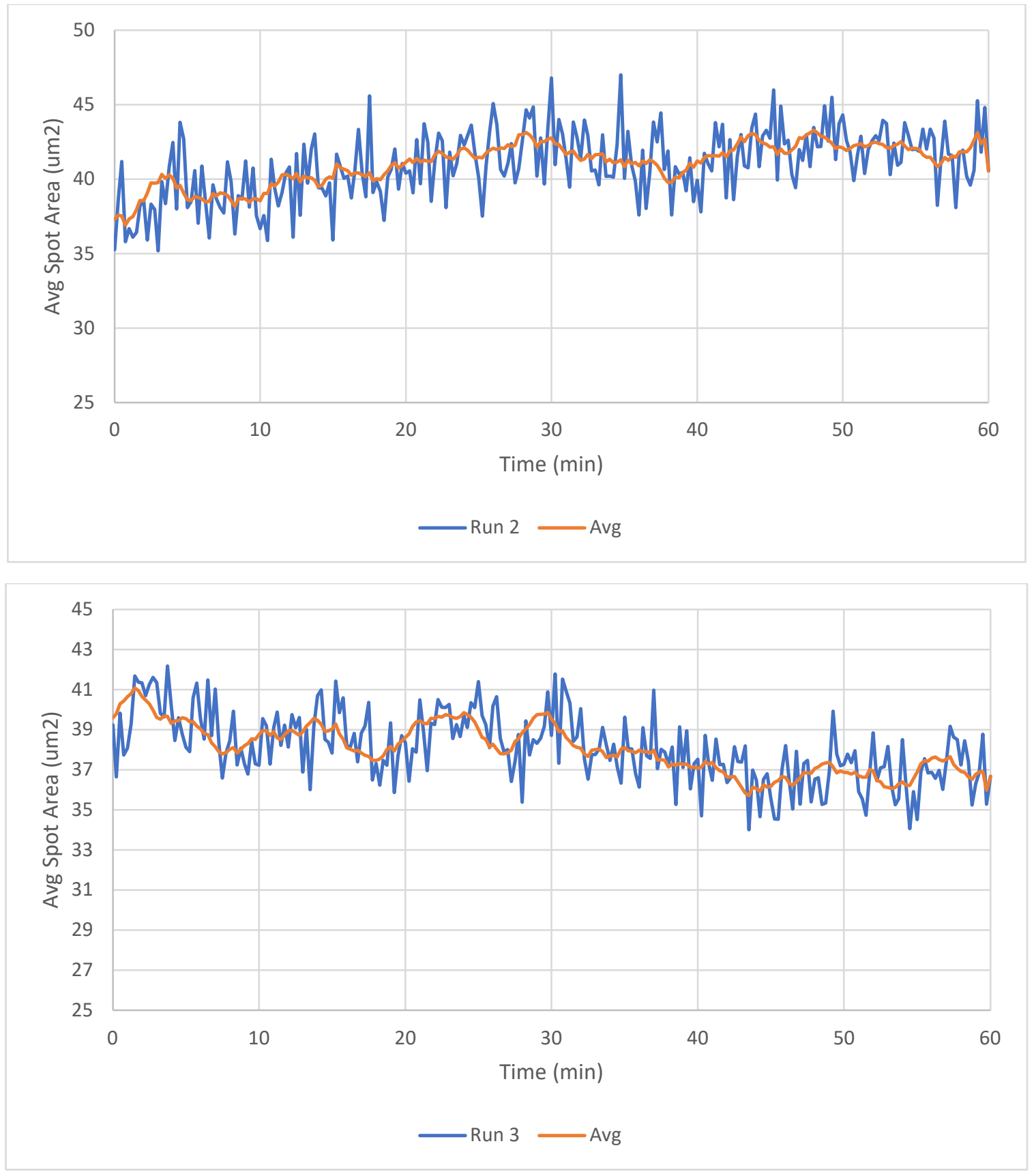


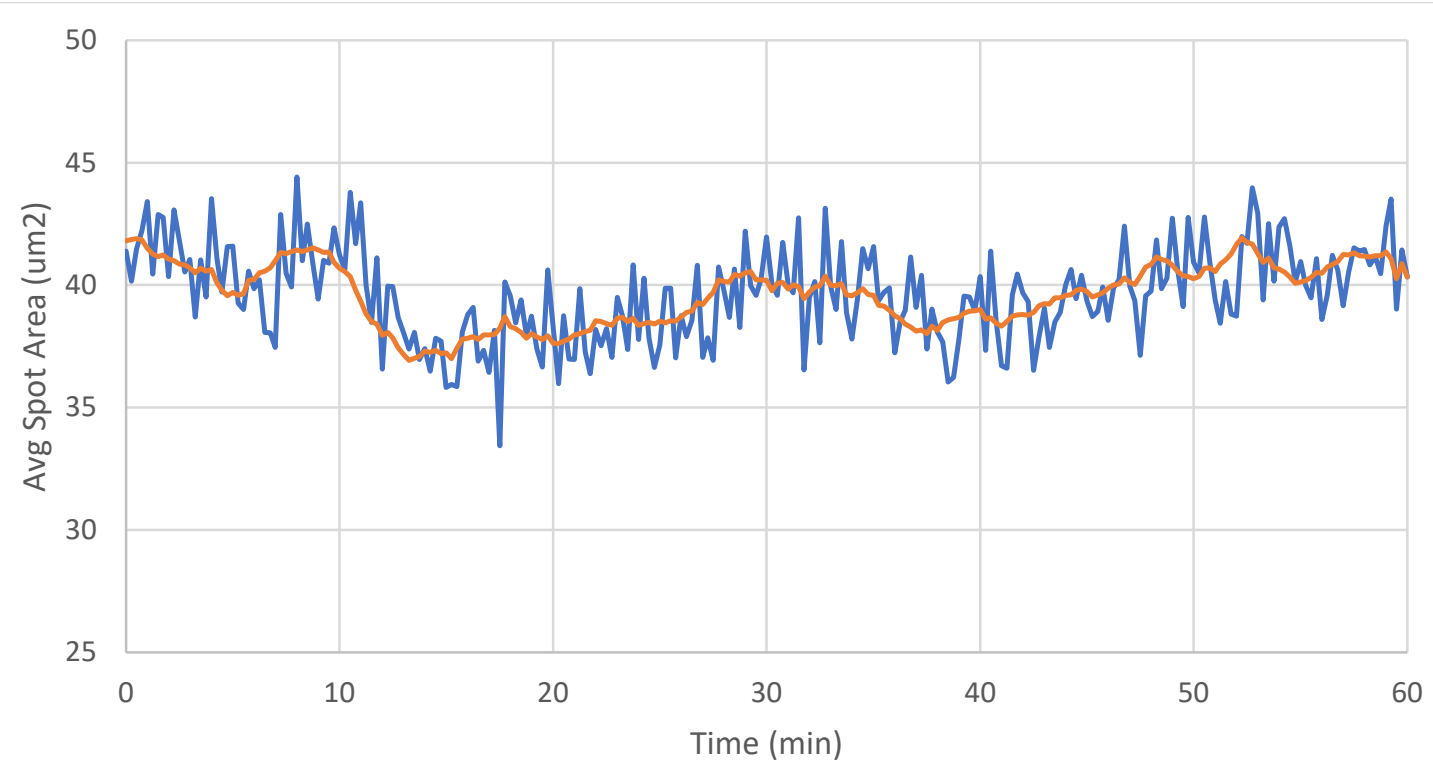

$\longrightarrow$ Run $4 \longrightarrow$ Avg

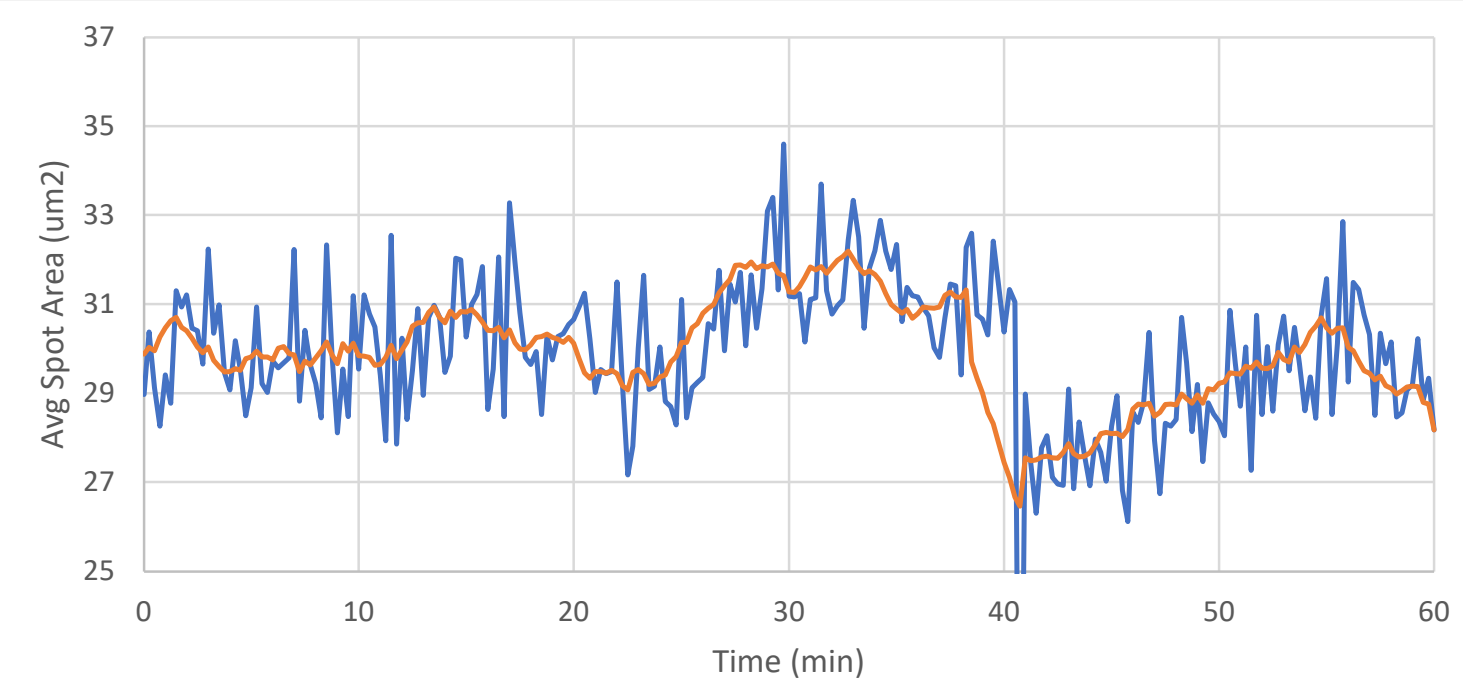

$\longrightarrow$ Run $5 \longrightarrow$ Avg 


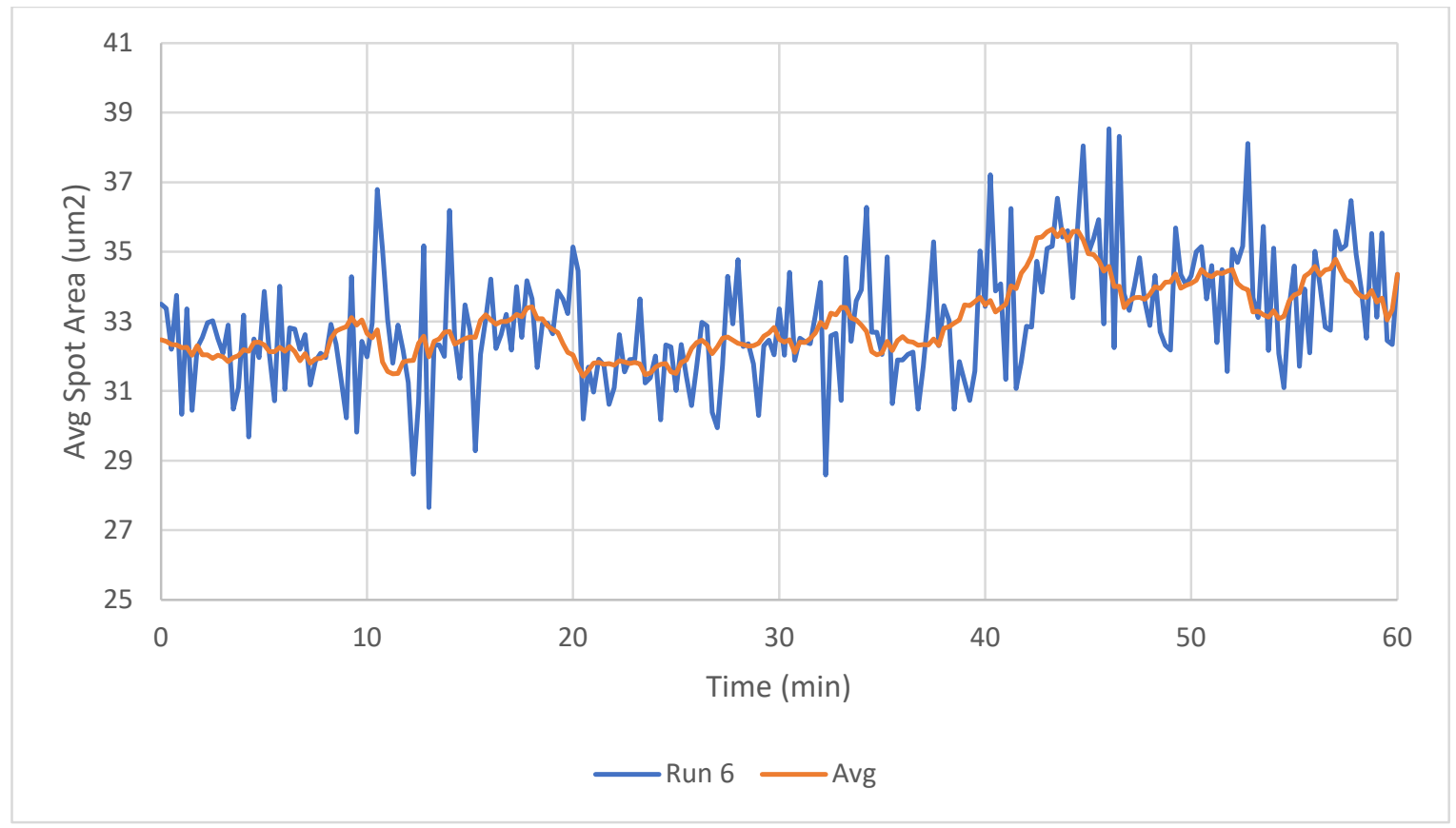

0.01 Volume Percent Bimodal Colloidal Mixture

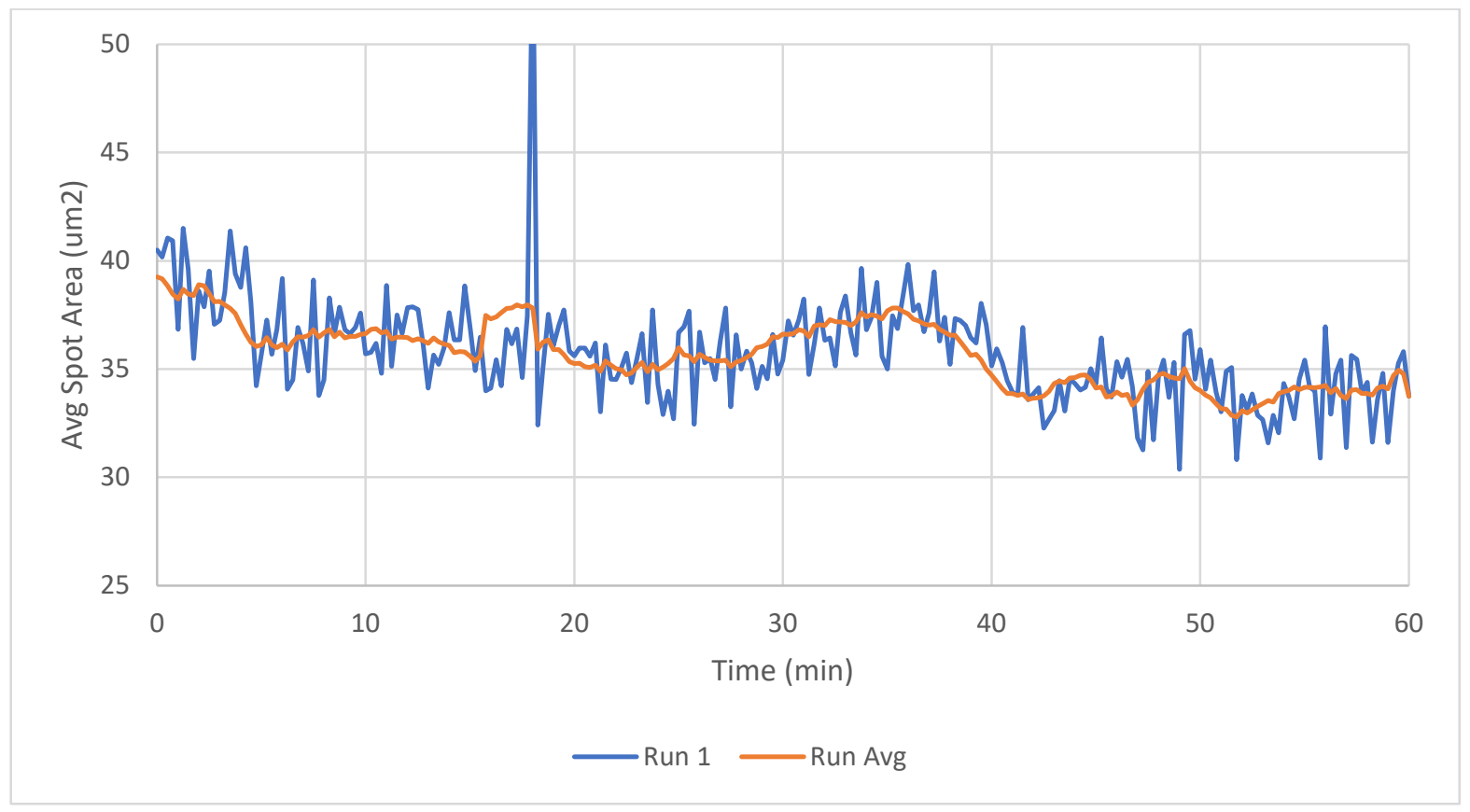



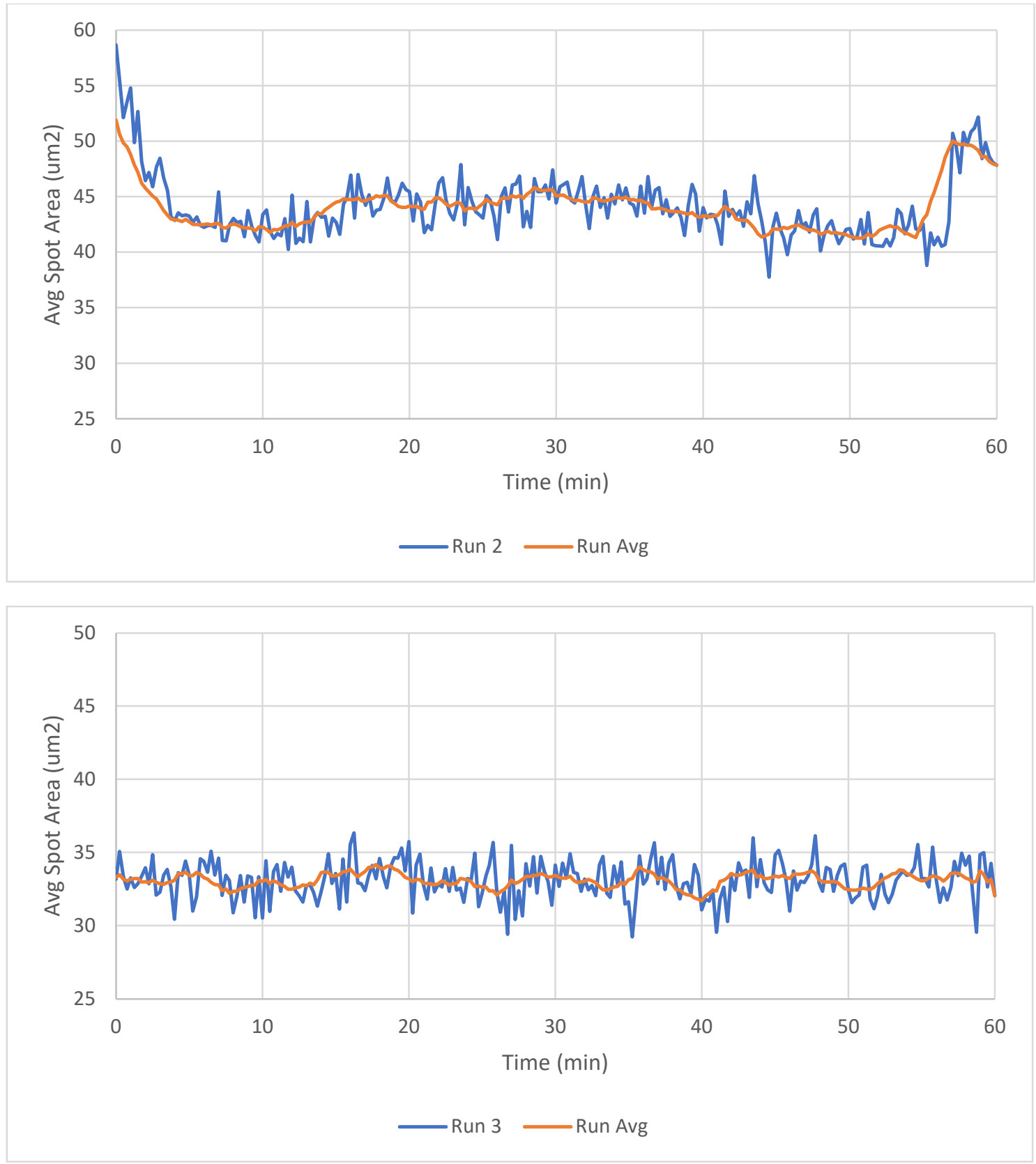


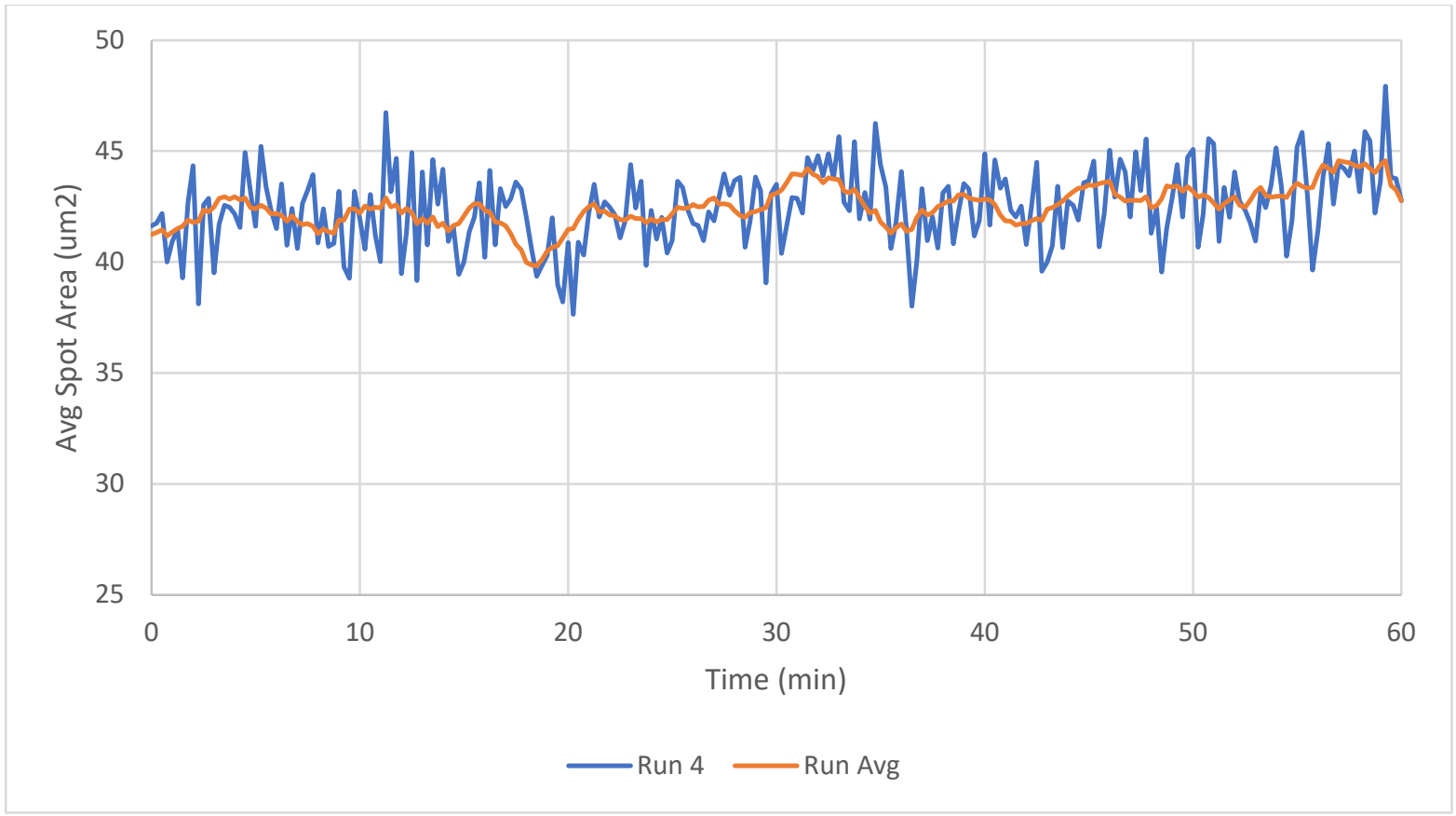




\section{APPENDIX III: FULL SOURCE CODE FOR BLOB SIZE ANALYSIS MACRO}

NASA Macro:

//Get path

input = getDirectory("Choose image file location");

list $=$ getFileList(input);

$/ / \operatorname{print}(\operatorname{list}[0])$;

first $=$ true;

setBatchMode(false);

for $(\mathrm{i}=0 ; \mathrm{i}<$ list.length; $\mathrm{i}++)\{$

path $=$ input + list $[\mathrm{i}]$;

showProgress(i, list.length);

if(!endsWith(path,"/")) open(path);

if(nImages $>=1)\{$

if(first) \{

waitForUser("Select area (rectangle) for analysis");

roiManager("Reset");

roiManager("Add");

close ();

first $=$ false;

setBatchMode(true);

open(path);

//Initialize table with path name

run("Table...", "name=Table width=350 height=250");

print("[Table]","\\Headings:FilenameNo. spots Spot area mean

Spot area SD T value $\quad$ Seconds since mix");

\}

roiManager("select", 0); 


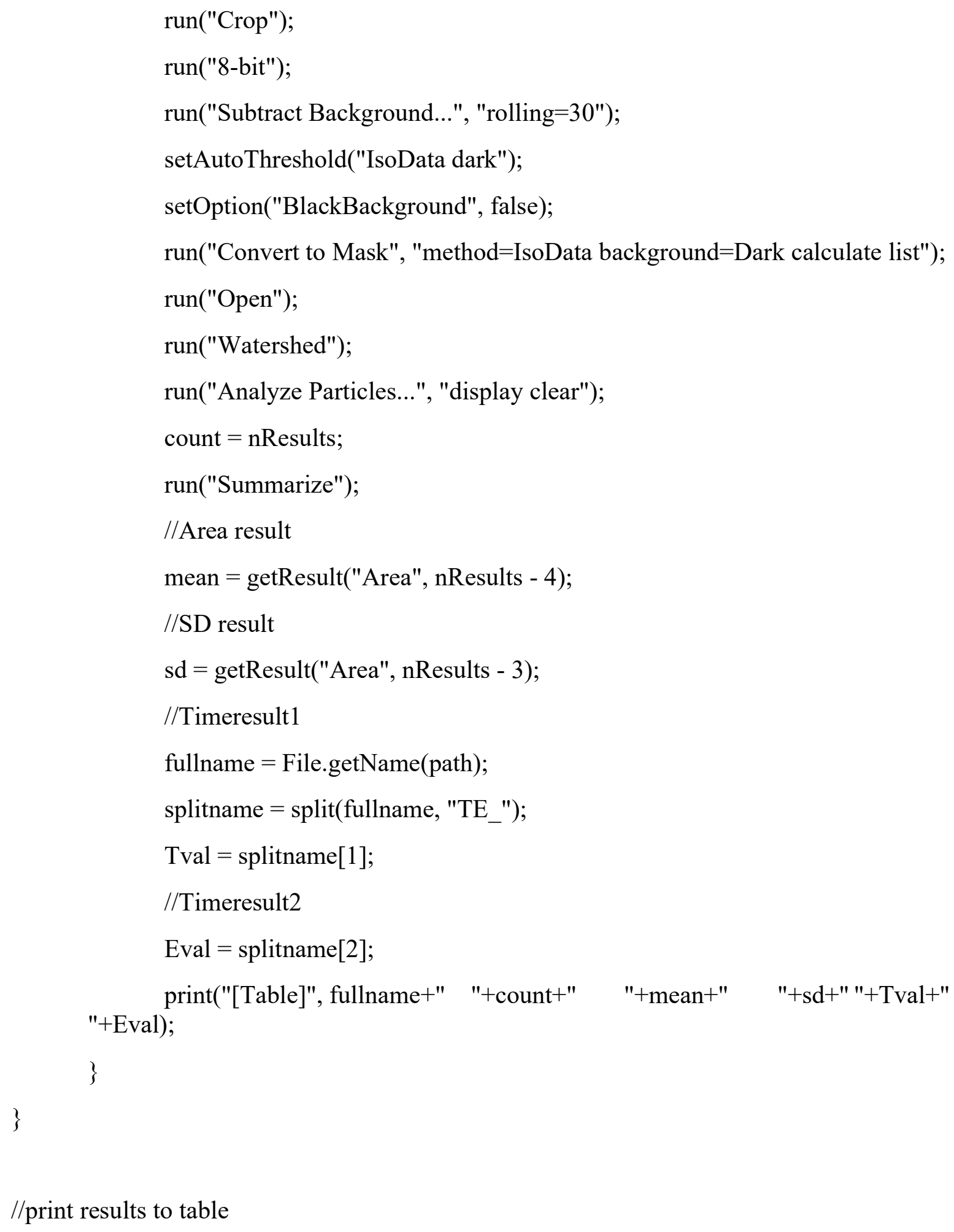




\title{
CURRICULUM VITA
}

NAME:

Luke Thomas Hawtrey

ADDRESS:

\author{
Department of Chemical Engineering \\ 216 Eastern Pkwy \\ University of Louisville \\ Louisville, KY 40208
}

DOB:

Lexington, Kentucky -January 17, 1996

EDUCATION \& TRAINING

B.S., Chemical Engineering

University of Louisville

$2015-2018$ 